\title{
New halo formation mechanism at the KEK compact energy recovery linac
}

\author{
Olga Tanaka, Norio Nakamura (中村 典雄), Miho Shimada (島田 美帆), \\ Tsukasa Miyajima (宮島 司), Akira Ueda (上田 明), \\ Takashi Obina (帯名 崇), and Ryota Takai (高井 良太) \\ KEK High Energy Accelerator Research Organization, \\ 1-1 Oho, Tsukuba, Ibaraki-ken 305-0801, Japan
}

(Received 3 July 2017; published 12 February 2018)

\begin{abstract}
The beam halo mitigation is a very important challenge for reliable and safe operation of a high-energy machine. A systematic beam halo study was conducted at the KEK compact energy recovery linac (cERL) since non-negligible beam loss was observed in the recirculation loop during a common operation. We found that the beam loss can be avoided by making use of the collimation system. Beam halo measurements have demonstrated the presence of vertical beam halos at multiple locations in the beam line (except the region near the electron gun). Based on these observations, we made a conjecture that the transverse beam halo is attributed to the longitudinal bunch tail arising at the photocathode. The transfer of particles from the longitudinal space to a transverse halo may have been observed and studied in other machines, considering nonlinear effects as their causes. However, our study demonstrates a new unique halo formation mechanism, in which a transverse beam halo can be generated by a longitudinal bunch tail due to transverse rf kicks from the accelerating (monopole) fields of the radio-frequency cavities. This halo formation occurs when nonrelativistic particles enter the cavities with a transverse offset, even if neither nonlinear optics nor nonlinear beam effects are present. A careful realignment of the injector system will mitigate the present halo. Another possible cure is to reduce the bunch tails by changing the photocathode material from the present GaAs to a multi-alkali that is known to have a shorter longitudinal tail.
\end{abstract}

DOI: 10.1103/PhysRevAccelBeams.21.024202

\section{INTRODUCTION}

The beam halo is a collection of low-density particles around a core of the beam distribution [1-2], originating from various sources. Its control is essential to attain highintensity beams. Therefore, experimental measurements and analytical evaluations of the beam halo distribution are very important to reduce particle losses, to minimize the radiation damage of accelerator components, and to lower the irradiation outside the machine, as well as to prevent cavity quenches. At the next linear collider (NLC), they impose very strict conditions for the collimation system to reduce the irradiation by the beam halo [3-4]. There are several mechanisms for the beam halo formation and resulting particle losses. They are associated with beam dynamics, design, errors, electron gun, vacuum system, etc. The beam dynamics issues include space charge effects [5], the Touschek, and the intrabeam scatterings [6-9]. The "design-related" mechanisms refer to the machine

Published by the American Physical Society under the terms of the Creative Commons Attribution 4.0 International license. Further distribution of this work must maintain attribution to the author(s) and the published article's title, journal citation, and DOI. nonlinearities and misalignments. Errors can be improper beam timing of laser or rf cavity phase shifts [10]. The vacuum system issues are residual gas scattering [11] and ion trapping. In addition to these well-known sources of halo formation, there are also some mechanisms unique to each machine that can generate beam halo. For comprehensive understanding of beam halo formation, systematic beam halo studies are very important.

At the compact energy recovery linac at KEK, our goal is to produce high-current and low-emittance electron beams (up to $10 \mathrm{~mA}$ ) without significant particle losses [12-14]. The maximum average current attained so far is about $1 \mathrm{~mA}$ at the beam energy of $20 \mathrm{MeV}$. It should be increased to $10 \mathrm{~mA}$ in a step-by-step manner during within a few years. At the current operation, the space charge has a negligible effect on the beam halo due to a relatively small bunch charge $(0.2-0.3 \mathrm{pC} / \mathrm{bunch})$. The effects of the longitudinal bunch tail produced at the photocathode are discussed in [15]. The other beam halo and particle loss mechanisms, such as the Coulomb scattering and the dark current, are studied in [16]. They are serious considerations in highenergy and high-intensity ERL operation, but for the present study with a low-intensity beam, they can be excluded from considerations. Typical parameters of the cERL are given in Table I. 
TABLE I. Typical parameters of cERL.

\begin{tabular}{lcc}
\hline \hline Parameters & Design & In operation \\
\hline Beam energy & $35 \mathrm{MeV}$ & $20 \mathrm{MeV}$ \\
Injector energy & $5 \mathrm{MeV}$ & $2.9-6.0 \mathrm{MeV}$ \\
Gun high voltage & $500 \mathrm{kV}$ & $390-450 \mathrm{kV}$ \\
Maximum beam current & $10 \mathrm{~mA}$ & $1 \mathrm{~mA}$ \\
Bunch length & $1-3 \mathrm{ps}$ & $1-3 \mathrm{ps}$ (usual) \\
& & $0.15 \mathrm{ps}$ (compressed) \\
Repetition rate & $1.3 \mathrm{GHz}$ & $1.3 \mathrm{GHz}$ (usual) \\
& & $162.5 \mathrm{MHz}$ (for LCS) \\
\hline \hline
\end{tabular}

Systematic beam halo studies have been conducted at the cERL since the machine commissioning in spring 2015. To understand beam halo formation processes at the cERL, we took some measurements during a machine study. In this study, we found some particle losses in the recirculation loop, when the beam passes through it without collimation. The observed particle losses are proportional to the bunch charge. This observation facilitates an investigation of the beam halo formation process. We activated COL1 and COL2 in the merger section (see Fig. 1), where the beam energy is low, to reduce the beam loss and to avoid the collimators activation. It worked for the beam loss mitigation in the recirculation loop. We also found that we can even enhance the beam loss reduction when the beam enters the injector cavities with a slight angle from the central axis of the injector [17]. This is achieved by inclusion of the steering coils of the injector line. Once a transverse beam offset is created and the off-center beam experiences rf field kicks, it could be cut away by the collimation system. Since no transverse beam halo has been observed at the electron gun vicinity, we conjecture that the driving mechanism of the beam halo formation is transfer of the longitudinal bunch tail into the transverse plane in the rest of the machine. The longitudinal bunch tail is assumed to be produced mainly by the cathode response on the laser excitation [18-19]. In this paper, we will show that beam halo simulations with this transfer mechanism can well reproduce observed beam halo profiles.

The injector part of the cERL is practically a linear system in the low-intensity operation. Nonlinear beam effects, such as the space charge, are negligible in the present low-intensity operation. In general, the phase space density of a beam must be preserved in a linear system (Liouville's theorem) and thus there should be no significant halo formation or emittance dilution at the cERL injector. Nevertheless, we have observed a non-negligible amount of particle losses at the cERL due to the halo. Many studies have been made to explain the halo formation or emittance dilution in a high-intensity operation [20-22] to overcome Liouville's theorem by considering nonlinear effects such as the space charge, coherent synchrotron radiation (CSR) effects, and diffusion effects (at JLab IR FER Driver, they achieved the total losses of less than $100 \mathrm{nA}$ around the entire machine by strictly controlling the halo [20]) or effects of input couplers in low-charge operation [23]. Apparently, these are not the case at the cERL injector in a low-intensity operation. Therefore, we had to find a new mechanism to explain the halo formation in the linear cERL injector. That is the motivation of this work.

We will summarize our findings in the next six sections. In Sec. II, a beam halo measurement, confirming the presence of halos, is described. Section III gives an analytical description of the longitudinal bunch tail generated at the photocathode. The transverse rf kicks at the injector cavities for nonrelativistic particles with transverse offsets are discussed in Sec. IV as a mechanism to enhance the transformation of the longitudinal bunch tail to the transverse halo. This effect turns out be essential in the present new halo formation study. The effect of steering coils on the beam trajectory is another important factor to enhance the beam halo formation. It is studied in Sec. V. Simulation results with all three effects combined are

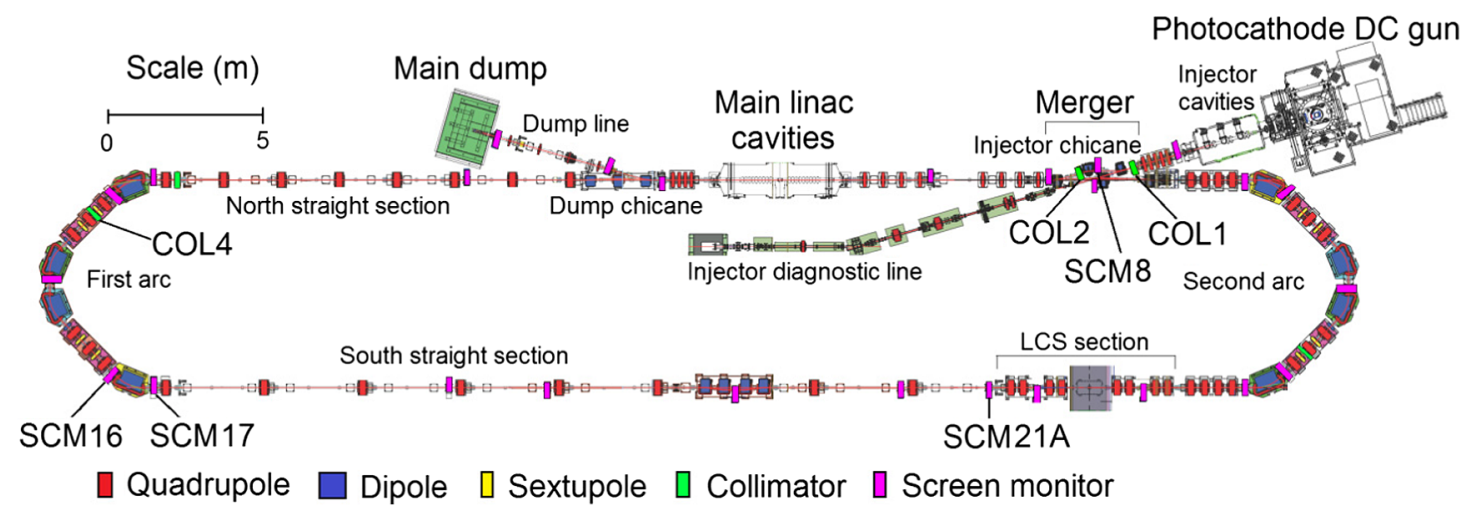

FIG. 1. Layout of cERL and locations of measurement equipment. The collimators COL1 and COL2 are located at the end of the injector before the first bending magnet, and between the second and the third bending magnets of the merger section, respectively. COL4 is located in the first arc section. The positions of screen monitors SCM8, 16, 17, and 21A are chosen to observe beam halo profiles in the merger section, at the first arc, at the exit of the first arc, and at the end of the south straight section, respectively. 


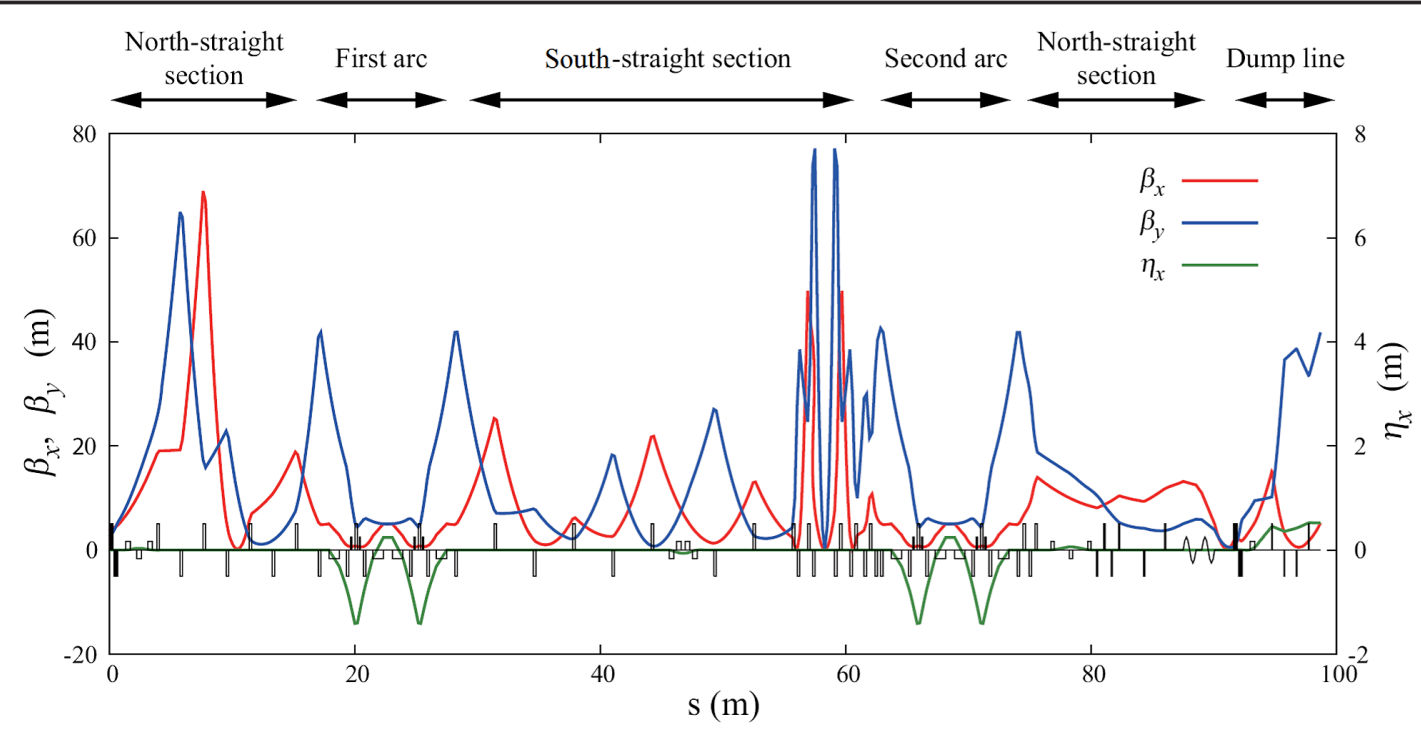

FIG. 2. Betatron and dispersion functions of the recirculation loop from the exit of the main linac cryomodule to the beam dump.

shown in Sec. VI. We consider also possibilities of the beam trajectory displacement due to some other effects at the injector cavities. Comparison with the measurements is presented. We also calculate particle losses due to the beam halo at various positions and compare them with measurements. The paper is concluded in Sec. VII.

\section{BEAM HALO MEASUREMENTS}

For observation of a vertical beam halo, we use several screen monitors (SCM), collimators in the merger section, and beam loss monitors to detect changes in the beam loss level during the measurement (see Fig. 1). The choice of the observation locations was made based on the radiation survey results [17]. Screen monitor SCM8 is positioned in the merger section between the two collimators COL1 and COL2 at the end of the injector where the dispersion may contribute to the halo formation. Screen monitor SCM16 is also located in the dispersive section at the end of the first arc. In these places, effects of the energy spread can be measured. The south straight section is known for particle losses [24]. Screen monitors SCM17 and 21A are positioned there. SCM17 is for detection of the beam profile in the place with large vertical beta function. The location of SCM21A in the south straight section [before the laser Compton scattering (LCS) section] is near the known loss point. The optical functions of the recirculation loop from the exit point of main linac cryomodule till the beam dump are shown in Fig. 2 [25].

A screen monitor system consists of a 12-bit CCD (charge-coupled device) camera (Allied Vision Technologies, Prosilica GC650) and a YAG:Ce screen. It is illustrated in Fig. 3 (top). A beam illuminates the YAG: Ce screen, mounted in a two-stage holder. Light from the screen, guided by a system of two mirrors, goes to the CCD camera. The geometric parameters of the screen monitor systems of screen monitors SCM8, 16, 17, and 21A are listed in Table II [26]. For the reader's reference, a more sophisticated beam halo measurement system can be found in Ref. [27].

It is known from beam operation experiences that the collimators COL1 and COL2 in the merger section are

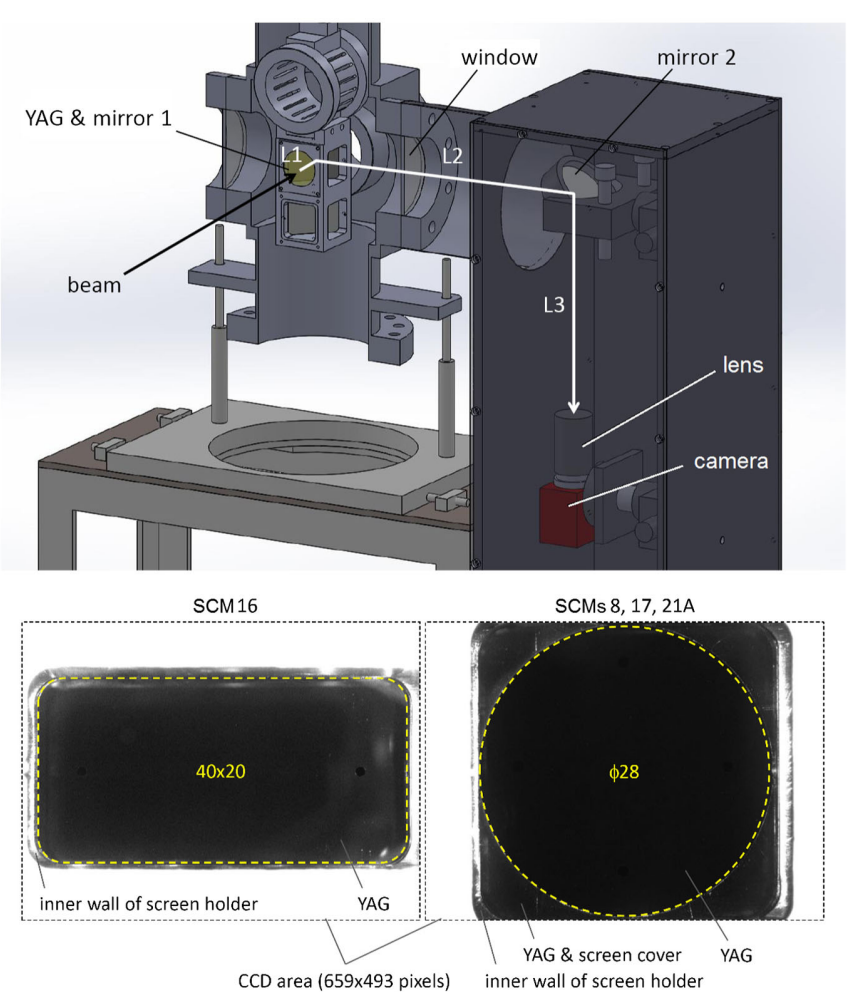

FIG. 3. Schematic of the screen monitor system with a 12-bit CCD camera and a YAG:Ce screen (top). Comparison of sizes of the YAG:Ce screens and the CCD camera screen images (bottom). 
TABLE II. Geometric parameters of the screen monitor systems.

\begin{tabular}{|c|c|c|c|}
\hline Parameters & SCM8 & SCM16 & SCM17, 21A \\
\hline YAG:CE screen size & $\phi 28 \mathrm{~mm}$ & $40 \times 20 \mathrm{~mm}$ & $\phi 28 \mathrm{~mm}$ \\
\hline $\mathrm{L} 1+\mathrm{L} 2+\mathrm{L} 3$ distances $^{\mathrm{a}}$ & $778 \mathrm{~mm}$ & $417 \mathrm{~mm}$ & $389 \mathrm{~mm}$ \\
\hline $\begin{array}{l}\text { Camera lens } \\
\text { Focal length } \\
\text { Aperture }\end{array}$ & $\begin{array}{c}100 \mathrm{~mm} \\
30 \mathrm{~mm}\end{array}$ & $\begin{array}{l}50 \mathrm{~mm} \\
28 \mathrm{~mm}\end{array}$ & $\begin{array}{l}50 \mathrm{~mm} \\
28 \mathrm{~mm}\end{array}$ \\
\hline
\end{tabular}

${ }^{\mathrm{a}}$ See Fig. 3.

effective to reduce particle losses in the recirculation loop. They are composed of two pairs of the horizontal and the vertical cylinder collimator jaws, whose gap and center are independently and remotely adjustable. The jaws are made of copper. The collimators have round beam ducts of $50 \mathrm{~mm}$ diameter (see Fig. 4) [28].

Beam halo measurements were performed for two settings of the operation modes (the burst mode and the long pulse mode) listed in Table III. Note than the long pulse mode gives the more distinct picture of the halo distribution; therefore, we choose such profiles for analysis. For SCM8 the data taken at the burst mode only is available. We allow the saturation of the core part (neutral-density (ND) filters taken out), because the shape of the halo can be captured clearly at the maximum gain.

Thus, vertical halos emerge at all of the camera locations. The measurement results are shown in Fig. 5: the left figures are when the collimators are taken out, while the right figures show when the collimators are inserted in. Measured beam halo profiles at a location of screen monitor SCM8 are taken at the burst mode with a gain of $22 \mathrm{~dB}$ and integration time of $10 \mu \mathrm{s}$, while those at the locations of screen monitors SCM16, 17, and 21A are taken at the long pulse mode with the same gain and integration time of $2 \mathrm{~ms}$. Basically, the vertical halos disappeared at all of the camera positions when collimators were inserted in.

Note that the light spots at the top and bottom of SCM16 profiles, and on the left and right of SCMs 8, 17, and 21A profiles are YAG screen lights reflected at the inner part of the screen holders and delivered directly to the CCD

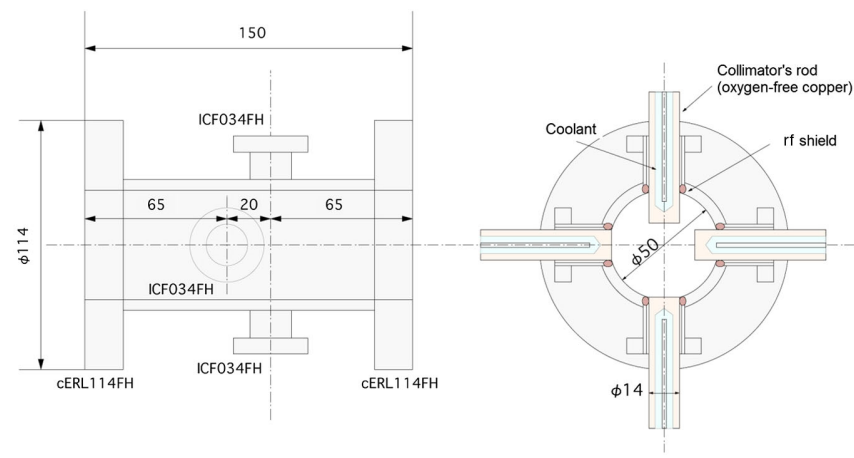

FIG. 4. Schematic of the collimators COL1 and COL2.
TABLE III. Settings for beam halo measurements.

\begin{tabular}{lcc}
\hline \hline Settings & Burst & Long pulse \\
\hline Macro pulse duration & $1 \mu \mathrm{s}$ & $1.5 \mathrm{~ms}$ \\
Macro pulse frequency & $5 \mathrm{~Hz}$ & $0.6 \mathrm{~Hz}$ \\
Exposure time of shutter & $10 \mu \mathrm{s}$ & $2 \mathrm{~ms}$ \\
Bunch charge & $0.2-0.3 \mathrm{pC}$ & $2.6 \mathrm{fC}$ \\
Average beam current & $1.5 \mathrm{nA}$ & $3 \mathrm{nA}$ \\
Peak beam current & $300 \mu \mathrm{A}$ & $15 \mathrm{nA}$ \\
Repetition rate & $1.3 \mathrm{GHz}$ & $1.3 \mathrm{GHz}$ \\
Beam energy & $2.9-20 \mathrm{MeV}$ & $20 \mathrm{MeV}$ \\
\hline \hline
\end{tabular}

camera's sensitive areas, whose sizes are larger than those of the corresponding screens (see the bottom figure of Fig. 3). These light spots should not be confused with diffraction at the camera's aperture (it is not allowed by the camera geometry) or with the CCD blooming caused by the CCD pixels saturation (otherwise halos will be seen regardless of presence of the collimators).

From these beam halo profile measurements, one can conclude that the collimation systems are effective to remove vertical beam halos. Another important finding is that the elimination of the vertical halo by the collimation system and the reduction of particle losses in the recirculation loop are synchronized. We believe it is a good indication of the effectiveness of the beam based tuning. We now have confirmed experimentally that there are nonnegligible transverse halos at the cERL. The next step is to find out their formation mechanisms, using the measurement data obtained. For this end, the following systematic study was performed.

\section{LONGITUDINAL BUNCH TAIL}

The measurement results demonstrate the presence of a spatial halo around a core of the beam. As mentioned in the Introduction, we suspect that the most likely cause of the beam halo at the cERL is the longitudinal bunch tail created at the photocathode, which is transferred into a transverse halo at the rest of the machine. Let us consider longitudinal bunch tail issues in details.

At the cERL a bulk $p$-type GaAs photocathode is utilized due to its ability to produce a low-emittance electron beam with high quantum efficiency [29]. The longitudinal time structure of the bunch depends on the response time of the cathode to the laser excitation. The response time is then determined by the process of internal transport of conduction electrons in the GaAs crystal. In the bulk GaAs cathode, electrons are generated deep inside of the GaAs crystal; therefore, it takes some time to travel to the crystal surface. The time response measurements of bulk GaAs photocathodes can be found in [30-31].

For longitudinal bunch tail simulations, we use the experimental data for the time response of the bulk GaAs photocathode at the laser wavelength of $520 \mathrm{~nm}$ 

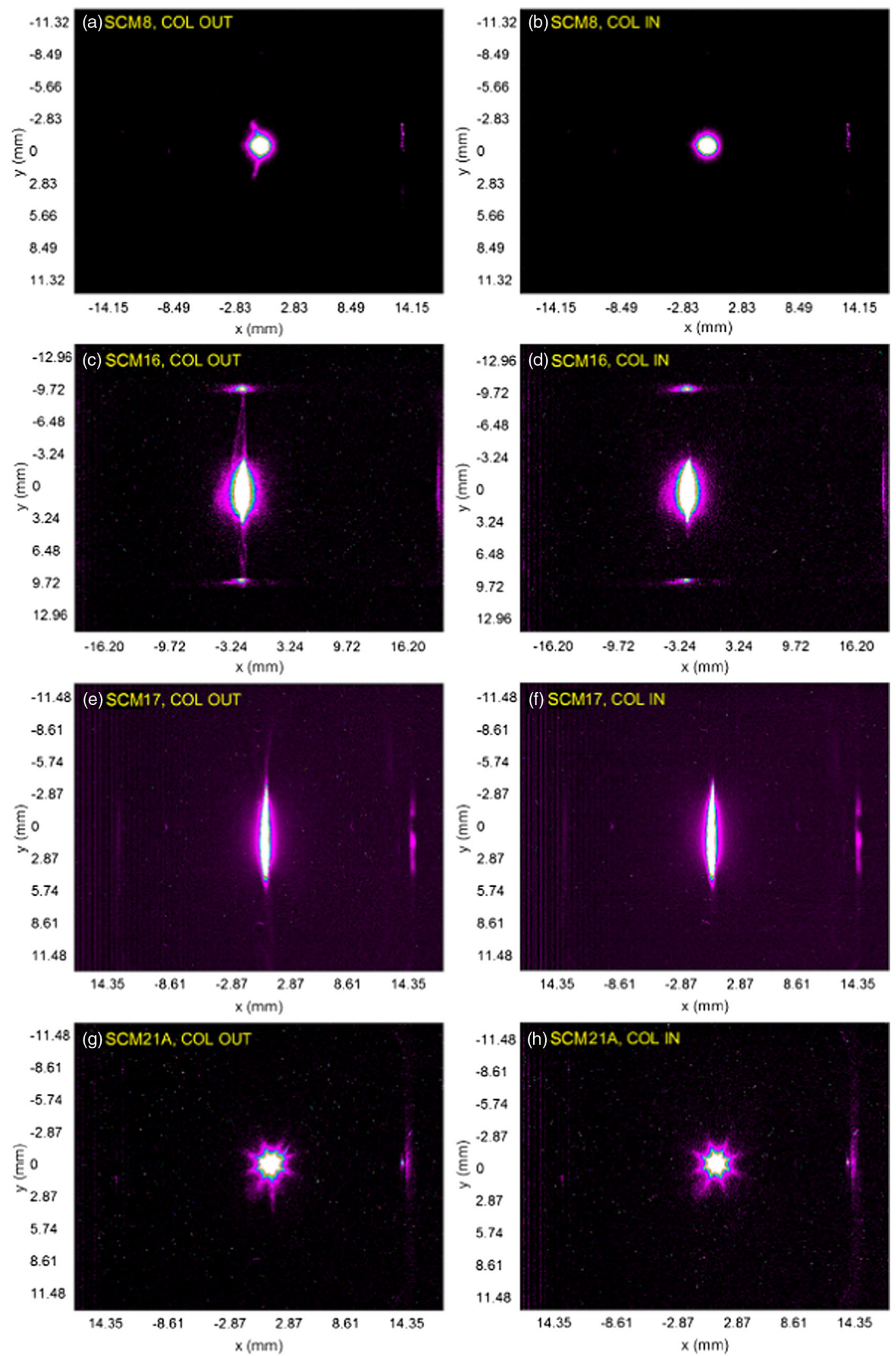

FIG. 5. Measured beam halo profiles at different beam line locations without (left) and with (right) collimation, respectively. (a),(b): Screen monitor SCM8 at the burst mode with gain $22 \mathrm{~dB}$ and integration time of $10 \mu \mathrm{s}$. (c)-(h): screen monitors SCM16, 17, and 21A at the long pulse mode with gain $22 \mathrm{~dB}$ and integration time of $2 \mathrm{~ms}$, respectively. 


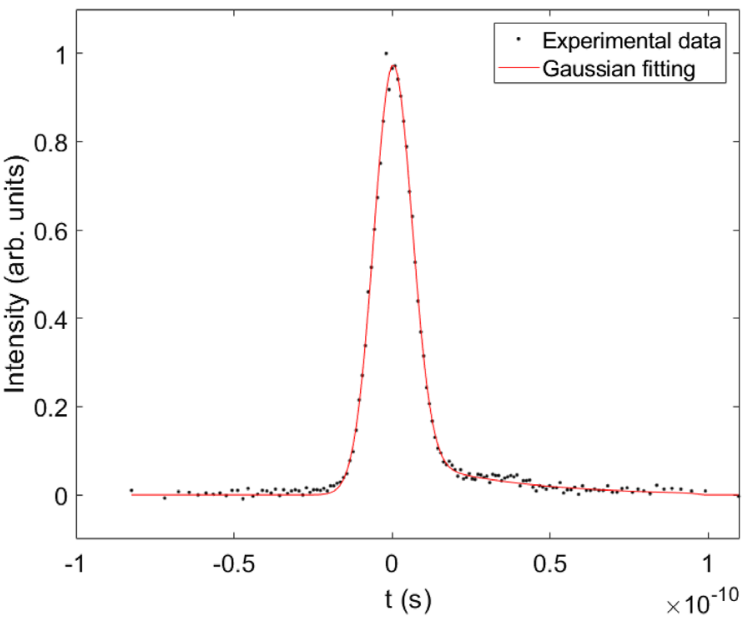

FIG. 6. Time response measurement of the bulk GaAs cathode at laser wave length of $520 \mathrm{~nm}$ (dots) by the projection of the bunch traces taken by the beam profile monitors. The solid line shows the fitting curve obtained by the least squares method using the model cathode response function.

(see [30] for the experimental data acquisition technique). Note that the laser pulse width is different from that of the cERL though the laser wavelength is the same. The projection of bunch trace intensities are plotted as dots in Fig. 6. The solid line in Fig. 6 denotes a fitting curve based on the method of the least squares (see Appendix A). A model function used in the fitting procedure is a convolution integral [32]:

$(f * g)(k)=\int_{-\infty}^{\infty} f(k) g(k-s) d k=\int_{-\infty}^{\infty} f(k-s) g(s) d s$

of the normal distribution, which comes from the laser pulse

$$
f(k)=\frac{1}{\sqrt{2 \pi \sigma^{2}}} e^{-\frac{k^{2}}{2 \sigma^{2}}},
$$

with the photoemission current function, introduced in [9]

$$
g(k) \propto \frac{1}{\sqrt{\pi k}}-e^{k} \operatorname{erfc}(\sqrt{k}) .
$$

Here $k=t / \tau$ is normalized time, $\tau=\alpha^{-2} D^{-1}$ is photoemission characteristic time, $D$ is the electron diffusion constant, and $\alpha$ is the optical absorption coefficient. The parameters $D, \alpha$, and, therefore, $\tau$ describe the nature of the cathode surface. For GaAs photocathode and laser wavelength of $520 \mathrm{~nm}, \tau \leq 1$ [18]. The photoemission current function is built on the basis of diffusion model (for details see, for example, [19]). The function erfc is the complementary error function [33]: $\operatorname{erfc}(x)=1-\operatorname{erf}(x)$, where $\operatorname{erfc}(x)=\frac{2}{\sqrt{\pi}} \int_{x}^{\infty} e^{-t^{2}} d t$,

Now, the convolution integral in Eq. (1) is evaluated numerically using matlab [34]. Its analytical evaluation is derived in Appendix B. The least squares fitting method gave the following values for the free parameters of the cathode response model function:

$$
\left\{\begin{array}{c}
\sigma=17.340 \mathrm{ps} \\
\tau=0.757 \mathrm{ps}
\end{array}\right.
$$

An important item to note is that we have evaluated photoemission characteristic time $\tau$, which is fixed for this cathode type and laser wavelength. The upper limit of the $\tau$ value for the GaAs photocathode and for the wavelength $520 \mathrm{~nm}$ is assumed to be 1 .

The next step is the construction of the longitudinal distribution for the proper laser excitation length. For the cERL it corresponds to $\sigma=3.3 \mathrm{ps}$ [35]. Thus, the probability density function of the longitudinal bunch size is a convolution of the $\sigma=3.3 \mathrm{ps}$ Gaussian signal with the cathode emission current function with $\tau=0.757 \mathrm{ps}$. The probability density function of the longitudinal bunch size is plotted in Fig. 7.

To introduce the longitudinal bunch tail into simulations, the initial longitudinal distribution of the beam was generated in accordance with the blue curve shown in Fig. 7. The cutoff of the longitudinal distribution was set to $100 \mathrm{ps}$. The initial transverse distribution is assumed to be uniform. Other input parameters for simulations are listed in Table IV. Then, the bunch distribution was tracked with General Particle Tracer code [36] through the accelerator lattice (from the electron gun to the exit point of the main

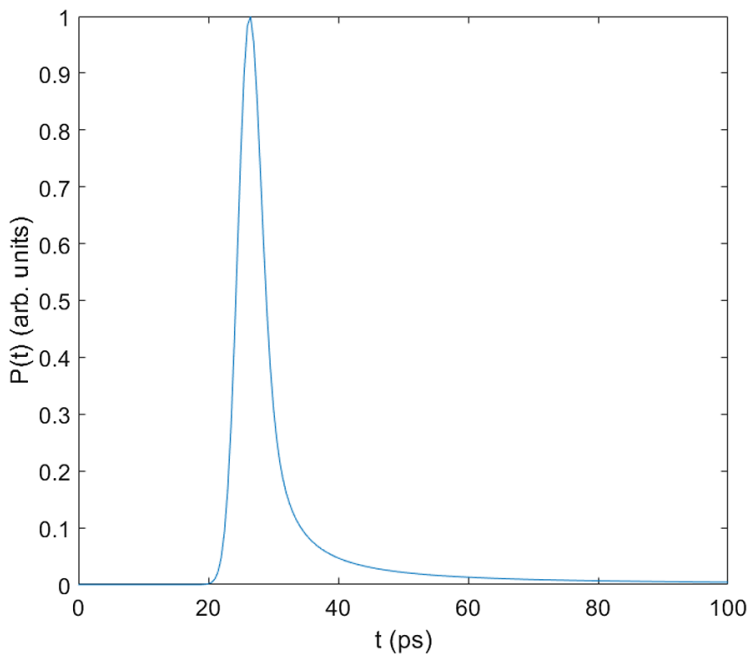

FIG. 7. Probability density function of the longitudinal bunch size obtained via convolution of the $\sigma=3.3$ ps Gaussian signal with the cathode emission current function with $\tau=0.757$ ps. 
TABLE IV. Input parameters for simulations.

\begin{tabular}{lcc}
\hline \hline Parameter & Value & Units \\
\hline Number of particles & $10^{4}$ & \\
Beam energy & $2.9-20$ & $\mathrm{MeV}$ \\
Total charge & 0.3 & $\mathrm{pC} / \mathrm{bunch}$ \\
Radio frequency & 1.3 & $\mathrm{GHz}$ \\
Laser spot diameter & 1.2 & $\mathrm{Mm}$ \\
Bunch length & & \\
Default & 3.3 & Ps \\
With tail & 100 & Ps \\
\hline \hline
\end{tabular}

linac cavities, see Fig. 1) to analyze the influence of the longitudinal bunch tail to the transverse beam profiles.

The results for the transverse beam emittances are presented in Fig. 8. Notice that the present definition of the emittance differs from the conventional one; it includes contributions from energy errors in the dispersive regions. One can clearly see that the horizontal emittance increases at the locations with nonzero dispersion such as the merger section (see Fig. 9). Betatron functions of the injector are shown in Fig. 10. Electrons at the Gaussian $\sigma=3.3$ ps core of the beam are accelerated on crest by the injector cavities up to energy $E=2.9 \mathrm{MeV}$. Electrons at the tail have a time retardation, and experience off-crest acceleration. Tail electrons exit the cavities with a large energy deviation of $\Delta E=0.64 \mathrm{MeV}$. The energy spread of electrons at the longitudinal tail results in a horizontal halo (from the lowenergy side) in the dispersive sections. This mechanism may explain the horizontal halo formation. However, the vertical dispersion exists only in the photocathode vicinity, while the large vertical halo is observed at screen monitor SCM8 where there is no vertical dispersion [see Fig. 5(a)]. Thus this mechanism alone (the transformation of the longitudinal bunch tail to a transverse halo through the dispersion) may not be sufficient to explain the vertical halo formation observed at the cERL. In the next section, we investigate transverse rf kicks in the cavities as an

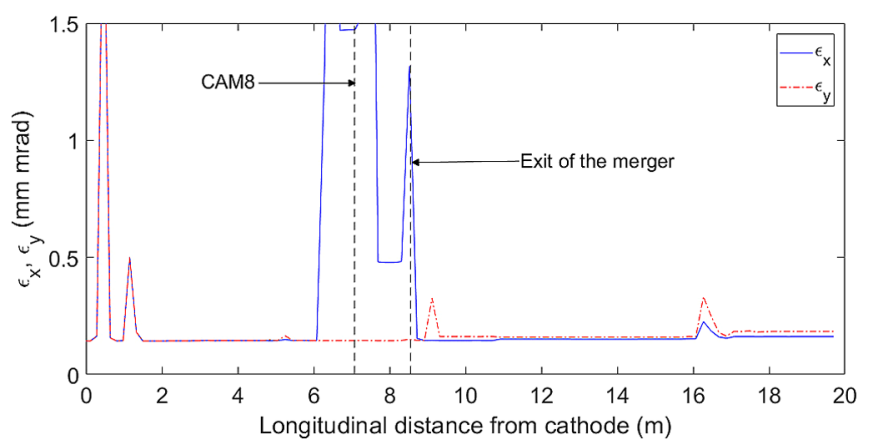

FIG. 8. The horizontal (solid line) and the vertical (dotteddashed line) emittances from the electron gun up to the exit of the main linac cavities for the Gaussian with $\sigma=3.3$ ps core with longitudinal tail $t_{\text {cutoff }}=100$ ps longitudinal distribution.

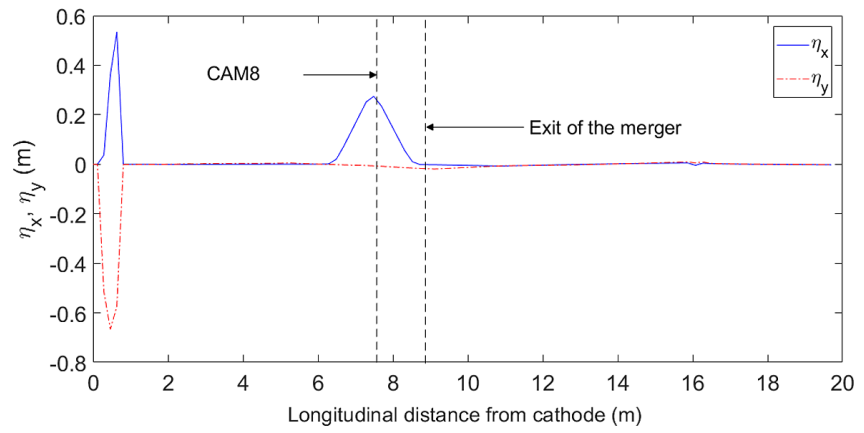

FIG. 9. The horizontal (solid line) and the vertical (dotteddashed line) dispersion functions from the electron gun up to the exit of the main linac cavities for the Gaussian with $\sigma=3.3 \mathrm{ps}$ core with longitudinal tail $t_{\text {cutoff }}=100 \mathrm{ps}$ longitudinal distribution.

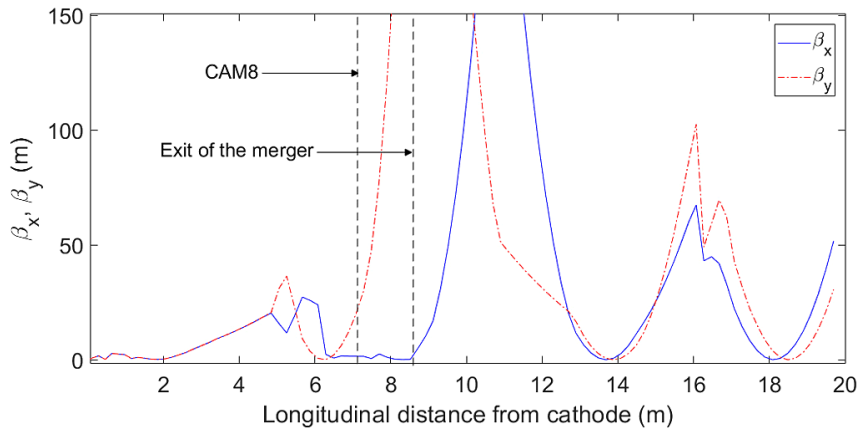

FIG. 10. The horizontal (solid line) and the vertical (dotteddashed line) betatron functions of the recirculation loop from the electron gun up to the exit of the main linac cavities.

enhancing effect on vertical halo formation in combination with the longitudinal bunch tail. For this end, we need to estimate the strength of rf kicks for various transverse offsets of the beam orbit inside the cryomodule.

\section{EFFECTS OF INJECTOR CAVITY RF KICKS}

The "design-related" mechanisms of the beam halo formation include misalignments of beam line elements, magnet field errors, and magnet noises [1]. Such mechanisms, especially in the linear transport line of the cERL, can lead to emittance growth and thus may enhance the beam halo at the same time. For example, we precisely adjusted the beam orbit in the injector solenoid to keep its axial symmetry. It helps to lower the emittance sufficiently [37]. However, it may not help to mitigate the transformation of the longitudinal bunch tail to the transverse halo. In order to examine this transformation mechanism, let us consider effects of injector cavity rf kicks.

The cryomodule at the cERL injector consists of three $1.3 \mathrm{GHz} 2$-cell superconducting cavities. The accelerating gradient was set to $6.42 \mathrm{MV} / \mathrm{m}$ for each cavity to deliver a 
2.9 $\mathrm{MeV}$ electron beam to the main linac [38]. Two input couplers of each cavity are intended to reduce a required rf power handling capacity, and to cancel out dipole kicks by the couplers. Each cavity has five loop-type higher order mode (HOM) couplers. Schematic of the cERL injector cryomodule is illustrated in Fig. 11.

It was hinted in a measurement [39] that the injector cavity misalignment is a possible cause of the beam halo. In this measurement, the nonuniformity of the relative positions of the three injector cavities was evaluated using the HOM coupler signals. It was found that the middle cavity has a $2.6 \mathrm{~mm}$ horizontal offset. No significant relative misalignments were found for the vertical offsets of the three cavities. However, there is a possibility that the entire cryomodule is shifted up or down, right or left due to some reasons such as cool-down cycles and long-term drifts. In this case, the three cavities are collectively shifted vertically or horizontally (this possibility is considered in Sec. VI). Such a collective shift was not measured, because the space is too tight to place the alignment tool due to the cERL beam line installation.

Particles can enter into the injector cavities with transverse offsets due to various mechanisms. The cavity misalignment can be translated to the radial displacement of a particle with respect to the cavity central axis. The steering coils can create transverse offsets of the beam trajectory more directly. Such displacement yields transverse kicks on particles moving inside the cavity. In addition, particles do not spend equal time at each cavity due to acceleration. They are essential effects in electron linacs [40]. The deflection of particle motion in rf cavities by transverse fields due to the orbit displacement is discussed in [41-43].

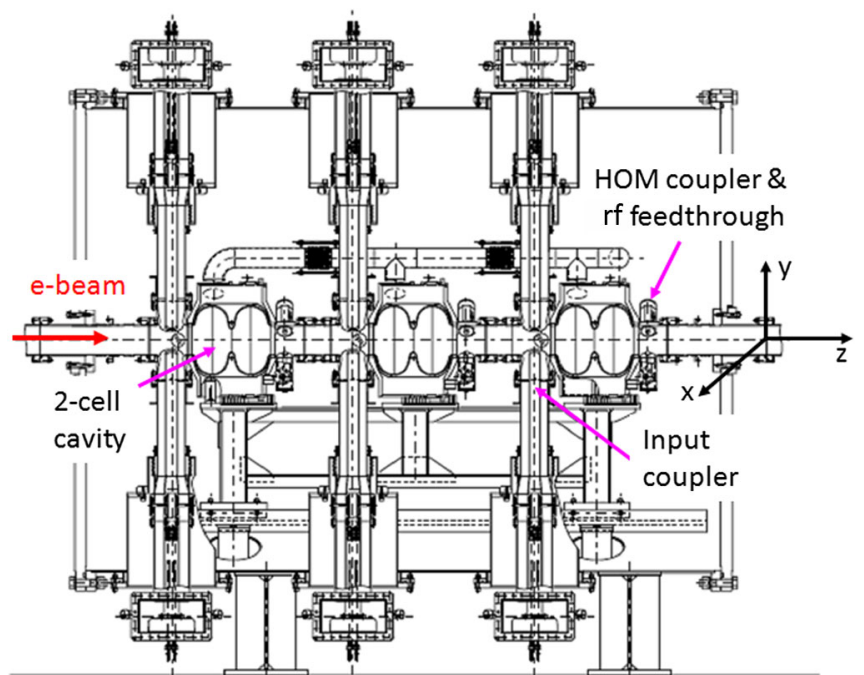

FIG. 11. Schematic of cERL injector cryomodule. The cryomodule includes three $1.3 \mathrm{GHz} 2$-cell superconducting cavities. Each cavity has two input couplers and five loop-type HOM couplers.
The nonzero transverse fields of the accelerating cavity $\pi$ mode, in terms of the cylindrical coordinates $(r, \theta, z)$, can be obtained from Maxwell's equations [40]:

$$
\begin{aligned}
\frac{\partial E_{r}}{\partial z}-\frac{\partial E_{z}}{\partial r} & =-\frac{\partial B_{\theta}}{\partial t} ; \quad-\frac{\partial B_{\theta}}{\partial t}=\frac{1}{c^{2}} \frac{\partial E_{r}}{\partial t} ; \\
\frac{1}{r} \frac{\partial\left(r B_{\theta}\right)}{\partial r} & =\frac{1}{c^{2}} \frac{\partial E_{z}}{\partial t} .
\end{aligned}
$$

The solution of Eq. (6) has a form

$$
\begin{aligned}
E_{z}(r, z, t)= & \frac{V_{0}}{2 \pi} \int_{-\infty}^{+\infty} d k[T(k) \cos k z \\
& +S(k) \sin k z]\left\{\begin{array}{l}
J_{0}(|K| r) \\
I_{0}(|K| r)
\end{array}\right\} \cos (\omega t+\phi) ; \\
E_{r}(r, z, t)= & \frac{V_{0}}{2 \pi} \int_{-\infty}^{+\infty} d k \frac{k}{|K|}[T(k) \cos k z \\
& -S(k) \sin k z]\left\{\begin{array}{l}
J_{1}(|K| r) \\
I_{1}(|K| r)
\end{array}\right\} \cos (\omega t+\phi) ; \\
B_{\theta}(r, z, t)= & -\frac{V_{0} \omega}{2 \pi c^{2}} \int_{-\infty}^{+\infty} d k \frac{1}{|K|}[T(k) \cos k z \\
& +S(k) \sin k z]\left\{\begin{array}{l}
J_{1}(|K| r) \\
I_{1}(|K| r)
\end{array}\right\} \sin (\omega t+\phi),
\end{aligned}
$$

where

$$
\begin{gathered}
V_{0}=\int_{-\infty}^{+\infty} E_{z}(r=0, z) d z, \\
T(k)=\frac{1}{V_{0}} \int_{-\infty}^{+\infty} E_{z}(r=0, z) \cos (k z) d z, \\
S(k)=\frac{1}{V_{0}} \int_{-\infty}^{+\infty} E_{z}(r=0, z) \sin (k z) d z, \\
K^{2}=\frac{\omega^{2}}{c^{2}}-k^{2} .
\end{gathered}
$$

The functions $J_{\alpha}(|K| r)$ and $I_{\alpha}(|K| r)(\alpha=0,1)$ are the Bessel function and the modified Bessel function, respectively. The choice of the function depends on the sign of $K^{2} \quad\left[J_{\alpha}(|K| r)\right.$ for $K^{2} \geq 0$, and $I_{\alpha}(|K| r)$ for $\left.K^{2} \leq 0\right]$. The parameter $k$ is the wave number coefficient, $k=\omega / c \beta=2 \pi f_{R F} / c \beta$.

To calculate a change in the transverse coordinate of a particle moving in the rf field, we first have to find out a change in the transverse momentum $p_{r}$. This change occurs due to the transverse component of the electric field 
$E_{r}(r, z, t)$ and azimuthal component of the magnetic field $B_{\theta}(r, z, t)$ :

$$
\Delta p_{r}=-q \int_{-\infty}^{+\infty}\left[E_{r}\left(r, z, t=\frac{z}{c \beta}\right)-c \beta B_{\theta}\left(r, z, t=\frac{z}{c \beta}\right)\right] \frac{d z}{c \beta} .
$$

Here, we assume that particles are moving in parallel to the $z$ axis ( $r=$ const, $z=c \beta t$ ), where $p_{r} \ll p_{z}$. We fix all the energy-dependent parameters like $\beta, \gamma, k$ as a constant and use their initial values in the evaluation of the integral (14). Following [44], the evaluation of Eq. (14) gives

$$
\begin{aligned}
\Delta p_{r}= & -\frac{q V_{0} \sqrt{k^{2}-k_{0}^{2}}}{c \beta k} I_{1}\left(\sqrt{k^{2}-k_{0}^{2}} r\right) \\
& \times[T(k) \sin \phi+S(k) \cos \phi] .
\end{aligned}
$$

Here $k_{0}=\omega / c$. To derive formulas for the $x$ and $y$ transformation, we use a normal vector $\mathbf{n}_{\mathbf{r}}=\left(x \mathbf{e}_{\mathbf{x}}+y \mathbf{e}_{\mathbf{y}}\right) / r$ $\left(\mathbf{e}_{\mathbf{x}}\right.$ and $\mathbf{e}_{\mathbf{y}}$ are the units vectors for the $x$ and $y$ directions, respectively) in the direction of the momentum kicks, so $\Delta \mathbf{p}=\Delta p_{r} \mathbf{n}_{r}$. Therefore,

$$
\left\{\begin{array}{l}
\Delta p_{x}=\Delta\left(\gamma \beta m c x^{\prime}\right)=m c(\gamma \beta)_{\text {out }} x_{\text {out }}^{\prime}-m c(\gamma \beta)_{\text {in }} x_{\text {in }}^{\prime} ; \\
\Delta p_{y}=\Delta\left(\gamma \beta m c y^{\prime}\right)=m c(\gamma \beta)_{\text {out }} y_{\text {out }}^{\prime}-m c(\gamma \beta)_{\text {in }} y_{\text {in }}^{\prime},
\end{array}\right.
$$

$$
\left\{\begin{array}{l}
x_{\text {out }}^{\prime}=\frac{(\gamma \beta)_{\text {in }}}{(\gamma \beta)_{\text {out }}} x_{\text {in }}^{\prime}-\frac{1}{(\gamma \beta)_{\text {out }}} \frac{x}{r} \frac{q V_{0}}{m c^{2} \beta \gamma} I_{1}\left(\sqrt{k^{2}-k_{0}^{2}} r\right)[T(k) \sin \phi+S(k) \cos \phi] ; \\
y_{\text {out }}^{\prime}=\frac{(\gamma \beta)_{\text {in }}}{(\gamma \beta)_{\text {out }}} y_{\text {in }}^{\prime}-\frac{1}{(\gamma \beta)_{\text {out }}} \frac{y}{m} \frac{q V_{0}}{m c^{2} \beta \gamma} I_{1}\left(\sqrt{k^{2}-k_{0}^{2}} r\right)[T(k) \sin \phi+S(k) \cos \phi] .
\end{array}\right.
$$

Equations (17) include the effect of energy gain inside the cavity. Note that they are valid only at low energy (low $\beta)$. The evaluation in the case of the axial symmetry is described in [45-46].

The physical understanding of the above formulas is clear. When a relativistic $(\beta=1)$ particle travels through the accelerating (monopole) mode of a cavity with a transverse offset, it should receive no transverse kick from the mode since kicks by the radial electric field and the azimuthal magnetic field cancel each other. That is not the case for a nonrelativistic particle with $\beta$ less than 1 , where the cancellation is not perfect and thus there is net transverse kick to a particle. In addition, particles receive different transverse kicks from the accelerating cavities depending on their relative longitudinal position from the core, since they enter the accelerating cavities at different rf phases. This effect is different from Rosenzweig and Serafini's cavity focusing effect [47] where they consider the fringe fields at the cavity entrance and exit for transverse focusing and the second-order ponderomotive focusing force from the cavity body proportional to the square of the field gradient for a relativistic particle. In our case, a nonrelativistic particle receives transverse kicks throughout the cavities due to imperfect cancellation of kicks by the radial electric field and the azimuthal magnetic field.

An analytical estimation based on the above model was performed for test parameters listed in Table V. Its result is shown in Fig. 12. The value of the transverse kick depends on the rf cavity phase $\phi$ and the $x_{\text {off }}$ or $y_{\text {off }}$ offsets inside the cavity. Thus, cavity kick simulations were carried out for different horizontal $(1,3,5 \mathrm{~mm})$ and vertical (fixed to $2 \mathrm{~mm}$ ) offsets. Note that the injector cryomodule have three cavities. The horizontal offset $x_{\text {off }}$ was applied only to the second cavity (in accordance with the measurement result [39]), while the same vertical offset $y_{\text {off }}$ was applied to the all three cavities simultaneously. The field maps of the injector cavities were calculated using poisson/superfish code [48]. The nonzero field components from the code output are plotted in Figs. 13-15. The phase offsets from the maximum gain of the first, second, and third cavities were set to $17.35^{\circ},-108.77^{\circ}$, and $71.16^{\circ}$, respectively. The simulated transverse cavity kicks $\Delta x^{\prime}$ and $\Delta y^{\prime}$ for various cavity offsets $x_{\text {off }}$ and $y_{\text {off }}$ are summarized in Table VI.

From these exercises, we have learned that a reasonable amount of the beam orbit displacement (a few $\mathrm{mm}$ ) inside the injector cavities can excite strong enough transverse $\mathrm{rf}$

TABLE V. Parameters of test calculations.

\begin{tabular}{lccc}
\hline \hline Parameter & Symbol & Value & Units \\
\hline Initial particle energy & $(\gamma \beta)_{\text {in }}$ & 1.4526 & \\
Final particle energy & $(\gamma \beta)_{\text {out }}$ & 5.5882 & \\
Initial particle velocity $^{\mathrm{a}}$ & & & \\
Horizontal & $x_{\text {in }}^{\prime}$ & $-4.079 \times 10^{-4}$ & $\mathrm{~m} \mathrm{rad}$ \\
Vertical & $y_{\text {in }}^{\prime}$ & $2.443 \times 10^{-3}$ & $\mathrm{~m} \mathrm{rad}$ \\
Trajectory offset & & & \\
Horizontal & $x_{\text {off }}$ & $1,3,5$ & $\mathrm{~mm}$ \\
Vertical & $y_{\mathrm{off}}$ & 2 & $\mathrm{~mm}$ \\
Accelerating gradient & $E_{\mathrm{accl}}$ & 6.42 & $\mathrm{MV} / \mathrm{m}$ \\
Cavity radio frequency & $f_{\mathrm{RF}}$ & 1.3 & $\mathrm{GHz}$ \\
\hline \hline
\end{tabular}

\footnotetext{
${ }^{\mathrm{a}}$ Normalized to the speed of light.
} 


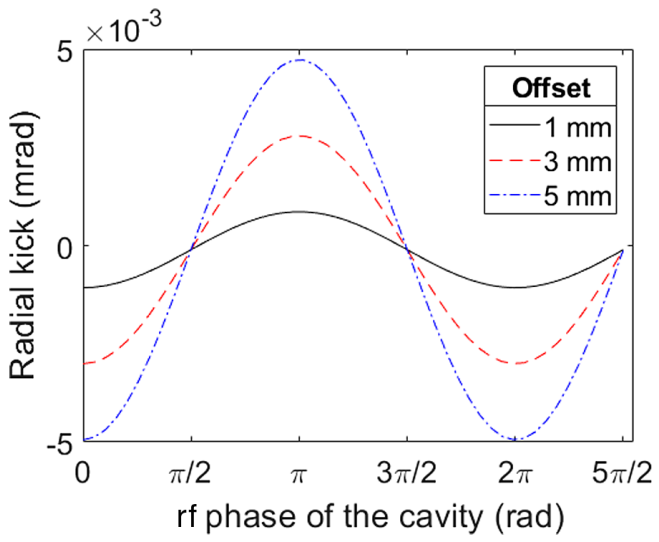

FIG. 12. Radial kick values for the horizontal trajectory offsets of $1 \mathrm{~mm}$ (solid line), $3 \mathrm{~mm}$ (dashed line), and $5 \mathrm{~mm}$ (dotteddashed), respectively, as a function of the cavity phase (the vertical offset is fixed to $2 \mathrm{~mm}$ ).

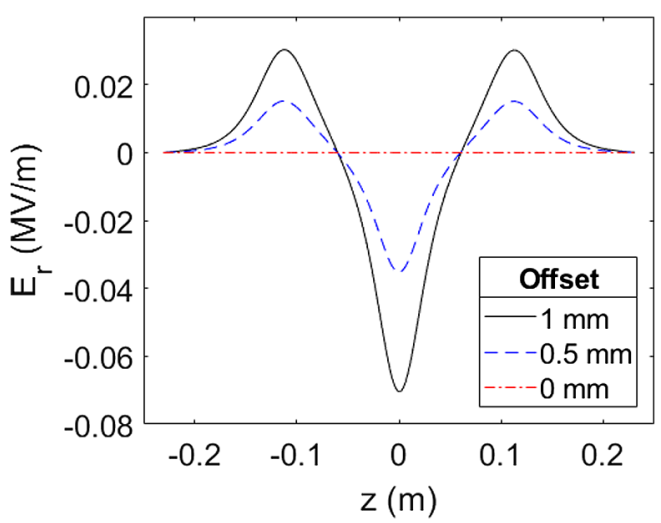

FIG. 13. Radial components of the electric fields in the cavities for different longitudinal offsets of $1 \mathrm{~mm}$ (solid line), $0.5 \mathrm{~mm}$ (dashed line), and $0 \mathrm{~mm}$ (on-crest acceleration) (dotted-dashed line), respectively.

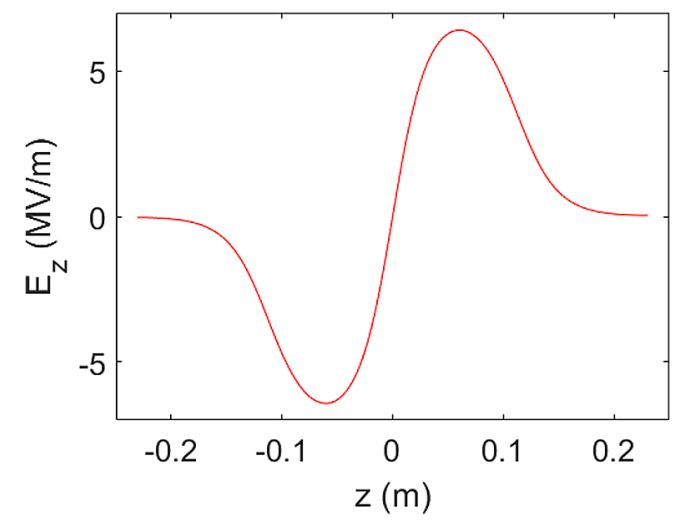

FIG. 14. Longitudinal component of the electric fields in the cavities.

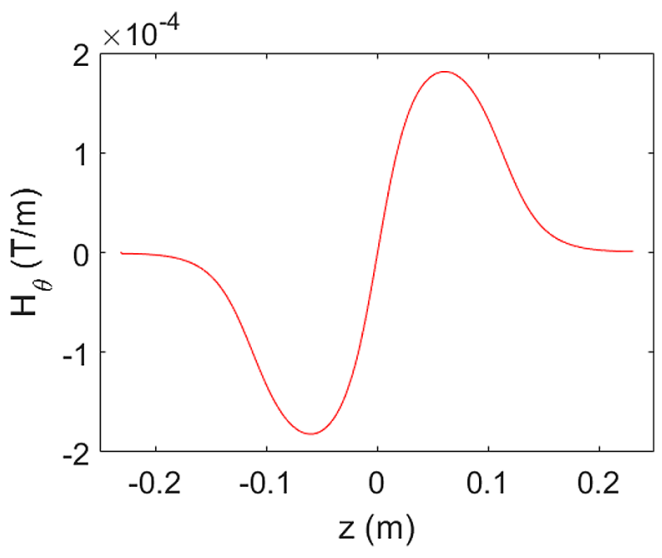

FIG. 15. Transverse component of the magnetic fields in the cavities.

kicks to particles, and the strength of those kicks largely depends on the particle's longitudinal position in a bunch. Particles in the core receive more or less a similar amount of transverse rf kicks from the cavity accelerating mode due to their vicinity in the longitudinal position. Therefore, they move together transversely. On the other hand, particles in the tail will receive quite different transverse rf kicks, sometimes even in the opposite direction, from those for the core, depending on their longitudinal distance from the core. Therefore, they start to deviate transversely from the core, creating a halo. This transformation of a longitudinal bunch tail to a transverse beam halo by the beam orbit displacement in a cavity can be a new mechanism of the halo formation.

The next step is to explore any possible factors of orbit displacements. The most probable factor is an influence of the injector line steering coils. In the next section we will investigate their effect on the beam trajectory.

\section{STEERING COIL EFFECTS ON BEAM TRAJECTORY}

Steering coils are indispensable devices for the orbit correction. As described in the previous section, when a beam enters the injector cavities with a transverse offset, it

TABLE VI. Simulated transverse cavity kicks $\Delta x^{\prime}$ and $\Delta y^{\prime}$ for various cavity offsets $x_{\text {off }}$ and $y_{\text {off }}$.

\begin{tabular}{lccc}
\hline \hline$x_{\text {off }}(\mathrm{mm})$ & $y_{\text {off }}(\mathrm{mm})$ & $\Delta x^{\prime}(\mathrm{mrad})$ & $\Delta y^{\prime}(\mathrm{mrad})$ \\
\hline 2.6 & 2 & -0.2916 & 0.2837 \\
2.6 & 0 & -0.2916 & 0.0018 \\
2.6 & -2 & -0.2916 & 0.2825 \\
0 & 2 & 0.0003 & 0.2837 \\
0 & 0 & 0.0003 & 0.0018 \\
0 & -2 & 0.0003 & 0.2825 \\
-2.6 & 2 & -0.2915 & 0.2837 \\
-2.6 & 0 & -0.2915 & 0.0018 \\
-2.6 & -2 & -0.2915 & 0.2825 \\
\hline \hline
\end{tabular}


experiences transverse rf kicks from the accelerating mode of cavities. Such a mechanism facilitates the formation of the transverse halo from the longitudinal bunch tail. Let us investigate effects of the injector steering coils on the beam trajectory in details.

There are two types of steering coils at the cERL injector. Those coils that are designated as "ZHV1-5" are a set of two pairs of rectangular Helmholtz coils [49] placed in parallel to the direction of beam propagation (see Fig. 16). A pair of horizontal coils is for vertical steering, and a pair of the vertical coils is for horizontal steering. Those named as "ZHV6-8" are two sets of two auxiliary coils wound on the poles of quadrupole magnets with a bore radius of $3 \mathrm{~cm}$ and core length of $10 \mathrm{~cm}$. The two separate sets of wires, one for the quadrupole and another for the steering coil, are wound on the same pole pieces. They have different numbers of turns and orientation. Therefore, they can be controlled independently. The schematic and simulated magnetic field lines of the auxiliary coils are shown in Fig. 17. The fields are uniform in the region around the central axis of the beam. However, a small amount of sextupole components arises due to the combination effect of the steering coil and the quadrupole. These fields are also included in the handling of the steering coils. The geometric parameters of the coils are listed in Table VII. Here the designations " $\mathrm{ZH}$ " and " $\mathrm{ZV}$ " correspond to the horizontal and the vertical steering, respectively. The magnetic fields of the pair of the rectangular coils are rather complicated [49-52]. Here, we apply the single loop approximation to describe the fields of the multiple turn coils. Such an approximation is valid if the cross section of the winding of a multiple turn coil is small compared to the

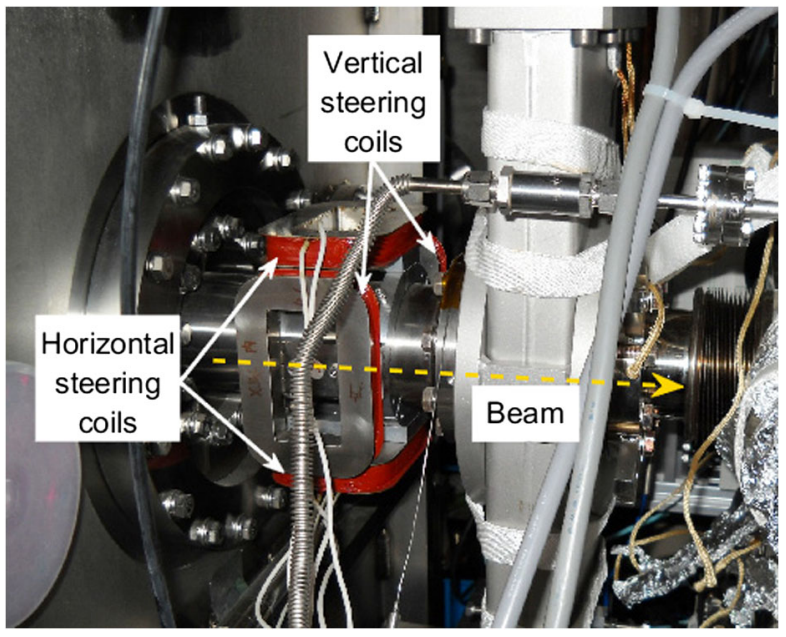

FIG. 16. Photograph of cERL injector steering coils. The electron beam travels from the left to the right. Two pairs of rectangular-shaped coils are placed in parallel to the beam line. A pair of rectangular-shaped coils on the left and on the right is used for vertical steering. Those at the top and at the bottom are for horizontal steering.

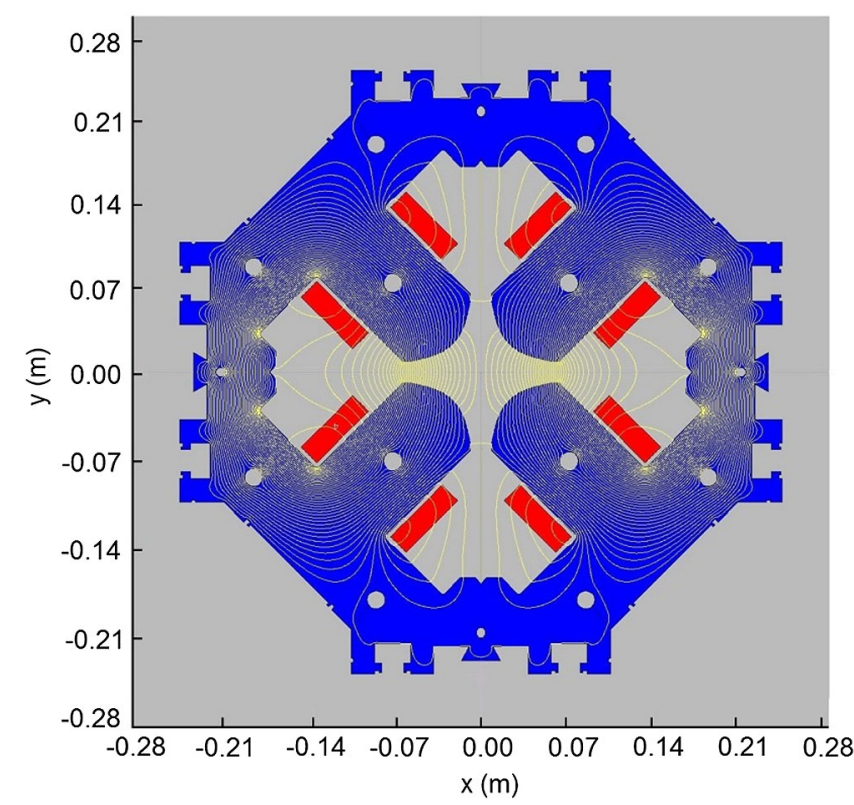

FIG. 17. Schematic of cERL injector steering coils wound on the poles of quadrupole magnets with the bore radius of $3 \mathrm{~cm}$ and core length of $10 \mathrm{~cm}$. Simulated magnetic field lines are also shown.

reach of the magnetic fields. See Appendix C for details on the magnetic flux calculation for the pair of rectangular loops. The axial components of the magnetic flux density vectors were evaluated numerically for each vertical steering coil. For the calculation of the magnetic field of the coils wound on the poles of the quadrupole magnets, we apply Opera simulation code [53]. Details on the field measurements of these magnets can be found in [54-55]. The magnetic fields of the steering coils are now included in computations of beam halo at the injector. The layout of the coils ZHV1-ZHV8 is shown in Fig. 18.

The total influence of the steering coils on the beam trajectory from the electron gun up to the entrance of the injector cavity was simulated. The result is shown in Fig. 19. The vertical offset $\Delta y$ is shown as a function of the longitudinal position $z$ when the steering coils are turned on and off. Note, here we neglect a slight difference between the shapes of the magnetic fields of square type and quad type coils. The magnetic field at the axis of the beam is supposed to be homogeneous. The Integral of magnetic fields $B_{x}(z)$ is equal to the increment of the beam tilt $\alpha$ [56]:

$$
\int_{0}^{z} B_{x}(z) d z=\frac{\gamma m c \beta_{z}}{e} \alpha=\frac{\gamma m c \beta_{z}}{e} \frac{\Delta y}{L} .
$$

Here $\gamma$ is the Lorentz factor, $m$ is electron mass, $c$ is speed of light, $\beta_{z}$ is a longitudinal component of the beam 
TABLE VII. Parameters of the injector line steering coils.

\begin{tabular}{|c|c|c|c|c|c|c|}
\hline Steering name & Current (A) & $\begin{array}{l}\text { Integrated field per current } \\
(\mathrm{T} \mathrm{m} / \mathrm{A}) \text { at the magnet center }\end{array}$ & Length (mm) & Gap (mm) & Width (mm) & Turns/coil \\
\hline ZH1 & -0.30 & $3.42 \times 10^{-5}$ & \multirow{2}{*}{59} & \multirow{2}{*}{133} & \multirow[b]{2}{*}{95.5} & \multirow{2}{*}{90} \\
\hline ZV1 & -0.90 & $3.23 \times 10^{-5}$ & & & & \\
\hline $\mathrm{ZH} 2$ & 0.06 & $5.93 \times 10^{-5}$ & \multirow{2}{*}{63} & \multirow{2}{*}{132} & \multirow{2}{*}{66} & \multirow{2}{*}{122} \\
\hline ZV2 & -0.18 & $6.07 \times 10^{-5}$ & & & & \\
\hline $\mathrm{ZH} 3$ & 0.00 & $5.93 \times 10^{-5}$ & \multirow{2}{*}{63} & \multirow{2}{*}{132} & \multirow{2}{*}{66} & \multirow{2}{*}{122} \\
\hline ZV3 & 0.00 & $6.07 \times 10^{-5}$ & & & & \\
\hline ZH4 & 0.71 & $3.21 \times 10^{-5}$ & \multirow{2}{*}{59} & \multirow{2}{*}{133} & \multirow{2}{*}{95.5} & \multirow{2}{*}{90} \\
\hline ZV4 & -3.18 & $3.57 \times 10^{-5}$ & & & & \\
\hline ZH5 & -0.82 & $7.07 \times 10^{-5}$ & \multirow{2}{*}{79} & 143 & \multirow{2}{*}{95.5} & \multirow{2}{*}{150} \\
\hline ZV5 & 0.25 & $7.48 \times 10^{-5}$ & & & & \\
\hline ZH6 & -4.90 & $1.83 \times 10^{-5}$ & \multirow[b]{2}{*}{100} & \multicolumn{2}{|c|}{ Bore radius } & \\
\hline ZV6 & 1.70 & $1.73 \times 10^{-5}$ & & \multicolumn{2}{|c|}{60} & 2 \\
\hline ZH7 & -0.43 & $1.83 \times 10^{-5}$ & \multirow{2}{*}{100} & \multirow{2}{*}{\multicolumn{2}{|c|}{60}} & \\
\hline ZV7 & 0.005 & $1.73 \times 10^{-5}$ & & & & 2 \\
\hline ZH8 & 0.00 & $1.83 \times 10^{-5}$ & \multirow{2}{*}{100} & \multirow{2}{*}{\multicolumn{2}{|c|}{60}} & \\
\hline ZV8 & -0.58 & $1.73 \times 10^{-5}$ & & & & 2 \\
\hline
\end{tabular}

velocity, $e$ is electron charge, and $L$ is the distance from the steering coil to the screen.

The simulation yields a small entry angle of $\alpha=0.138^{\circ}$ to the injector cavities from the central axis of the injector and a vertical offset of $\Delta y=1.69 \mathrm{~mm}$ at the first cavity location. In the next section we will summarize our investigations on the beam halo formation mechanisms and will present simulation results when all three effects (the longitudinal bunch tail, the rf kicks in the injector cavities due to beam orbit displacement, and the steering coils' influence on the beam trajectory) are put together. We also show simulation results of particle losses at the various camera positions and compare them with the measurement results.

\section{SIMULATIONS WITH THREE EFFECTS COMBINED TOGETHER}

Let us summarize the beam halo mechanisms investigated in the previous sections and see how they can reproduce the measured vertical beam halos if all three effects are combined.

\section{A. Qualitative treatment}

The first step is to find a right combination of the halo formation factors, which reproduces well the measured profile of the vertical halo. The main difficulty was to reach the halo distribution close to the one observed during the measurement. If one considers the longitudinal bunch tail alone, only one part of halo distribution (upper or lower, depending on the observation point location) can be reproduced. For example, for screen monitor SCM8, the longitudinal bunch tail causes the lower part of the halo. For this end we reexamined in detail the data of the cathode response measurement (see Sec. III). Upon closer examination a small percentage of particles (about $1.5 \%$ of the beam) outstripping the beam core in time was detected (see Fig. 6). The value of $1.5 \%$ was evaluated by the ratio of the area under the curve (Fig. 20) with the area under the Gaussian $(\sigma=3.3 \mathrm{ps})$ curve. The Gaussian area is assumed to be $100 \%$ and represents a core of the distribution in Fig. 6. Non-Gaussian particles can exist if the laser pulse profile is slightly different from the Gaussian one, or the stray light from the laser system arrives slightly earlier at the cathode than the main laser pulse. We then fit the
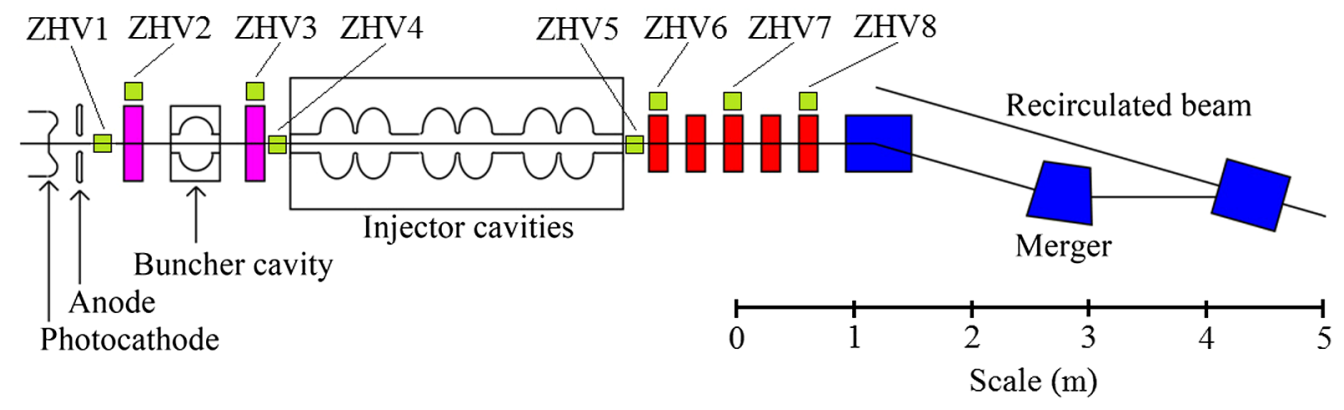

$\square$ Steering coil $\square$ Solenoid $\square$ Quadrupole $\square$ Dipole

FIG. 18. Layout of steering coils ZHV1-ZHV8 in cERL injector line (before the main linac starts). 


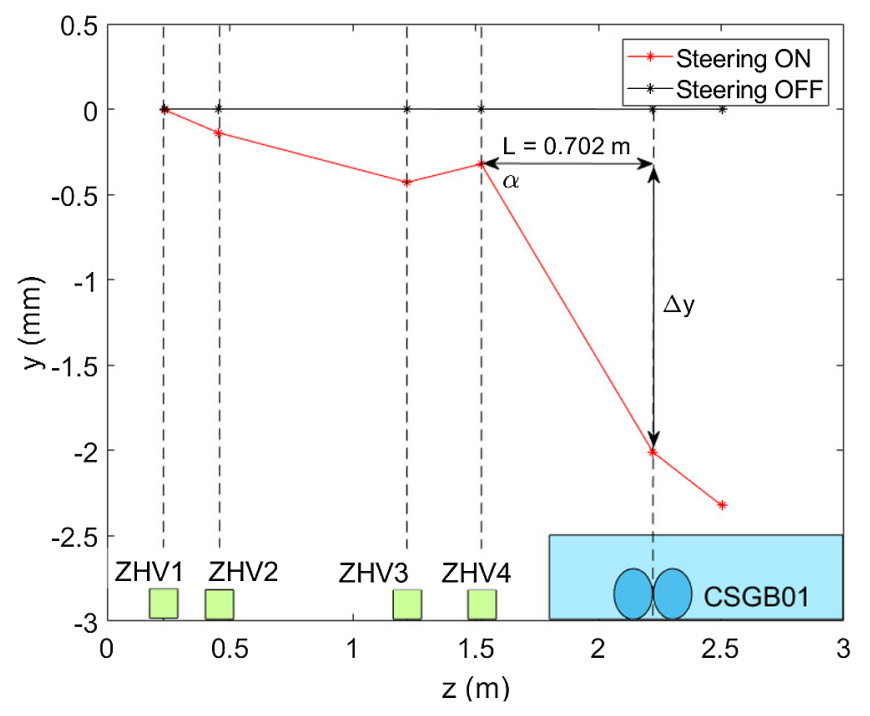

FIG. 19. Influence of the steering coils on the electron beam trajectory. The trajectory is shown on the $z-y$ plane. It is simulated for two cases: when all coils are turned on (dotted-dashed line) and off (solid line), respectively. The total effect of ZHV1-ZHV4 on the beam trajectory is a vertical offset of $\Delta y=1.69 \mathrm{~mm}$ at the first injector cavity location. The corresponding entry angle is $\alpha=0.138^{\circ}$.

corresponding data points properly and add this tiny forward tail (Fig. 20) to the longitudinal distribution. It greatly affects the halo simulation results. Then, for an observation point at screen monitor SCM8 location, the upper part of the beam halo appeared [see Fig. 21(c)].

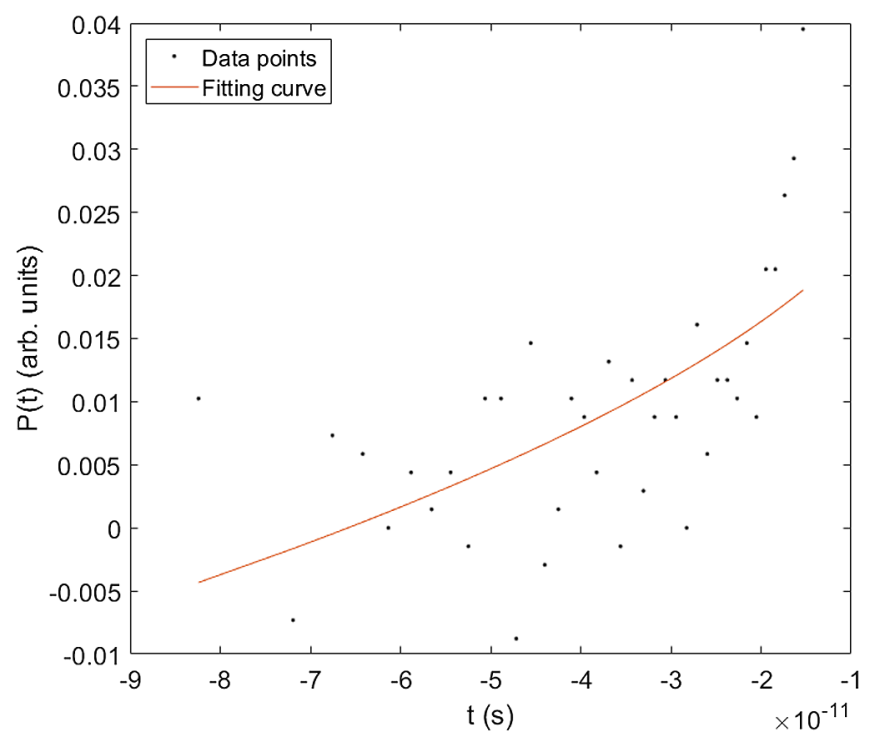

FIG. 20. Time response measurement results of the bulk GaAs cathode at laser wavelength of $520 \mathrm{~nm}$ in the interval from $-100 \mathrm{ps}$ to $-10 \mathrm{ps}$ (dots). The solid line shows the fitting curve obtained by the least squares method using the model function. The area under the fitting curve is about $1.5 \%$ of the total area of the longitudinal beam distribution.
Figures 21(a) and 21(b) show the transverse beam profiles without bunch tail and with only back tail, respectively. It is clearly seen that the beam halo comes from the bunch tails, not the core, and inclusion of both the back and forward tails are essential for accurate simulations.

Parameters of some devices that create transverse beam offsets in the injector cavities are already known. The currents values of the steering coils ZHV1-8 are known from the operation log. The relative horizontal offset of the injector cavity no. 2 is measured to be $2.6 \mathrm{~mm}$ [39]. As possible other effects for the beam orbit displacement, we can think of (i) collective displacements of all the three cavities in the horizontal and/or the vertical directions and (ii) ambient magnetic fields at the cERL. The ambient magnetic fields can arise due to geomagnetism or other unexpected local sources of a magnetic field. It is extremely difficult to measure or estimate. Therefore, let us simply consider that this effect is already included in (i). We should emphasize that basic injector cavities can produce significant energy deviation along the longitudinal distribution of the bunch by the acceleration field and transfer the longitudinal bunch tail into the transverse halo by transverse rf kicks. Therefore, it is essential to consider the beam orbit displacement inside the cryomodule and assume that there are additional beam orbit displacements there (on top of the steering coil effect), notably due to the collective cavity offset and possibly due to the ambient magnetic fields.

Let us use the collective cavity offset as a free parameter in simulations and find the optimum value that reproduces the measured beam halo profiles at the different locations (as shown in Fig. 5) well. If that happens, that collective offset may not be entirely inconceivable. The values $-2,0$, and $2 \mathrm{~mm}$ were tested for the collective horizontal and vertical offsets of cavities no. 1 through no. 3. Simulations were performed in the presence of a relative horizontal offset of $2.6 \mathrm{~mm}$ of injector cavity no. 2 and the longitudinal bunch distribution with the back and forward tails. All the steering coils were turned on, and the collimators COL1 and COL2 were taken out. The simulation results of the beam halo profiles at the screen monitor SCM8 location are shown in Fig. 22 for various values of the vertical offset. The different values of the horizontal offset have little effect on the vertical halo formation, as can be easily expected. Comparing the simulated halo profiles (Fig. 22) with the measured one [Fig. 5(a)] at screen monitor SCM8, it is easy to see that Fig. 22(c) reproduces the measured profile [Fig. 5(a)] remarkably well. Thus, one can conclude that it is very likely that a collective vertical offset of the beam trajectory, due the misalignment of the injector cryomodule, or due to the ambient magnetic fields, exists and it is about $2 \mathrm{~mm}$, though it is very difficult to confirm it experimentally at the cERL (the optical measurement system was removed before installation of the recirculation loop). 

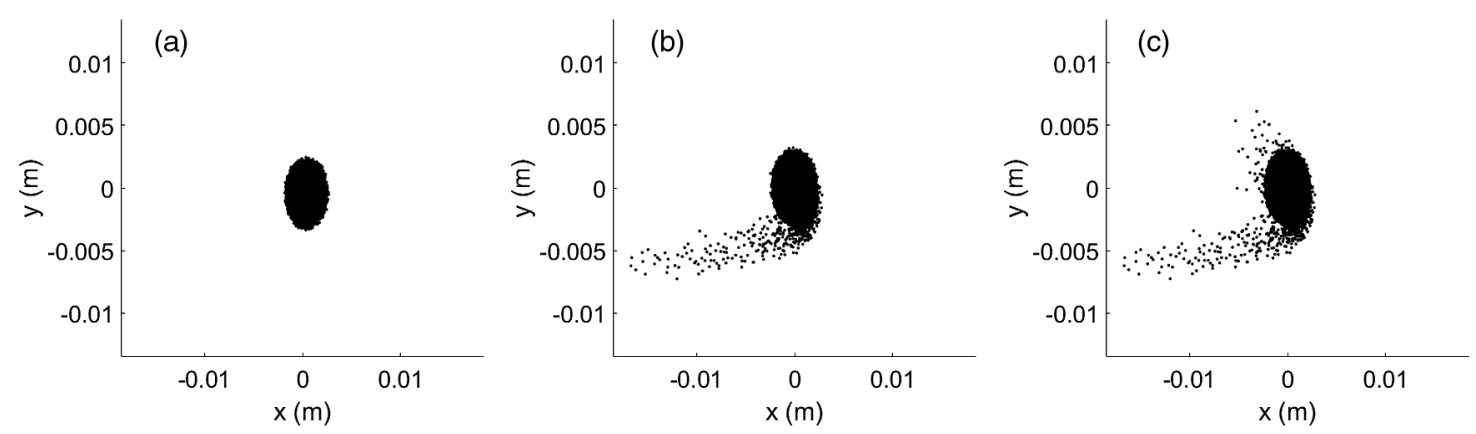

FIG. 21. Simulated beam halo profiles at screen monitor SCM8 location. The steering coils are on. The horizontal offset of the cavity No. 2 is $2.6 \mathrm{~mm}$, and the common vertical offset of $2 \mathrm{~mm}$ is assumed for the cavities Nos. 1-3. The collimators COL1 and COL2 are taken out. (a) Demonstration of those for the 3.3 ps Gaussian core. (b) For the 3.3 ps Gaussian core with 100 ps bunch back tail. (c) For the 3.3 ps Gaussian core with back and forward tails.
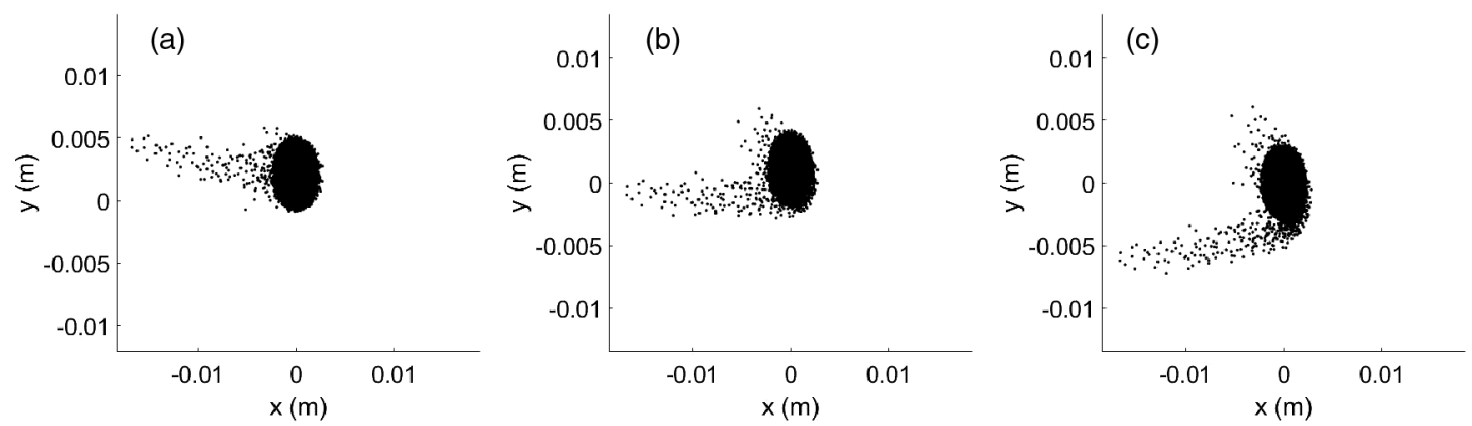

FIG. 22. Simulated beam halo profiles at screen monitor SCM8 location. Horizontal offset of the cavity No. 2 is 2.6 mm. The longitudinal bunch distribution includes $3.3 \mathrm{ps}$ Gaussian core, and the back and forward tails. The steering coils are turned on. The collimators COL1 and COL2 are taken out. (a) Demonstration of those for the collective vertical offset of cavities Nos. 1-3 of -2 mm. (b) For those with the collective vertical offset of $0 \mathrm{~mm}$. (c) For the offset $2 \mathrm{~mm}$.

One more important finding is that the effects of steering coils' and cavities' misalignments almost compensate each other in the horizontal direction, while in the vertical direction they add up. Therefore, off-crest particles of the beam distribution experience more transverse kicks than ones on crest, when propagating inside the cavity via off-axis trajectory. This mechanism causes the vertical beam halo, as observed in the measurements.

Now, all the simulation parameters are fixed, and we can move on to beam halo simulations at various screen monitors positions: $8,16,17$, and $21 \mathrm{~A}$. The simulations from the exit of the main linac cryomodule to the beam dump (see Fig. 1) are conducted with elegant tracking code [57]. For the complete comparison with the measured profiles shown in Fig. 5, we also turned on and off the collimators COL1 and COL2. The final simulation results for beam halo profiles are shown in Fig. 23. Although there are still some quantitative discrepancies between the measured profiles (Fig. 5) and the simulated ones (Fig. 23), the overall qualitative similarities are striking. This may imply that our understanding of beam halo formation mechanisms at the cERL and the simulation techniques are close to the reality.

From the simulation results, we learned that it is likely that the upper part of the halo at SCM8 comes from the particles of the forward tail, while the lower part comes from the back tail. At SCMs 16, 17, and 21A, the effects are reversed; the upper part is due to the back tail and the lower part is due to the forward tail.

\section{B. Quantitative treatment}

Now that the qualitative description of the beam halo formation has been made, we seek more quantitative agreements between the measurements and the simulations. For this end, we compare the particle population at the core and the halo in the measurements and the simulations at the various screen monitor positions. This procedure requires the following: (1) The simulations show that the transverse beam profile becomes bell shaped as a beam goes through many components. On the other hand, the measured signals from all the screen monitors saturate at the core area due to lack of the dynamic range. As a result, the measured beam profiles look like a rounded trapezoidal shape with a flat top. Thus, we fit both sides of the beam profiles (not the core) by a Gaussian distribution for every slice of the measured data captured. (2) Integration over the fitting curve of every slice of the capture [summation of the bins of every stroke of the 2D histogram (for the simulated profiles)] and summation over all the slices [strokes (for 

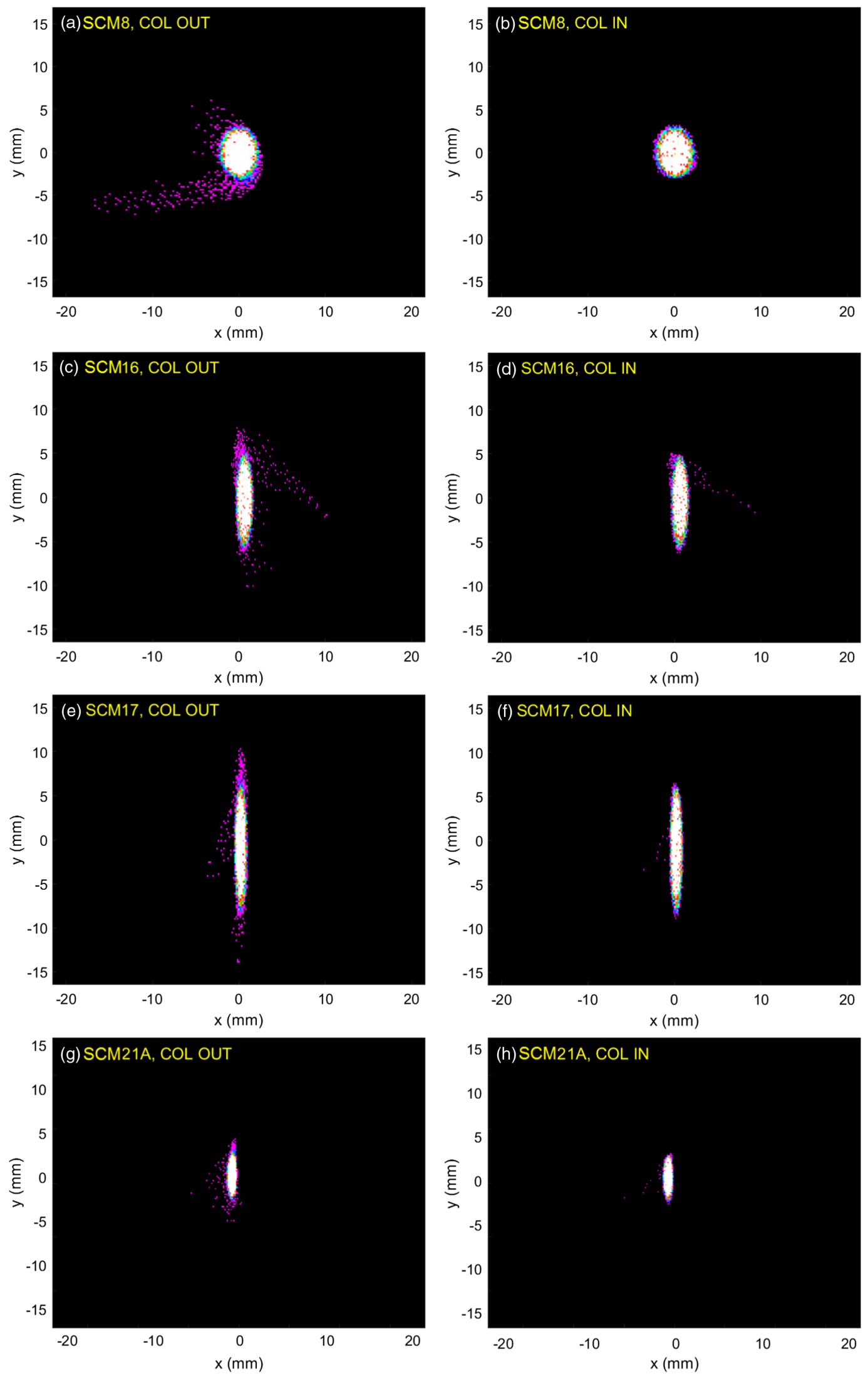

FIG. 23. Simulated beam halo profiles at different screen monitor locations without (left) and with (right) collimation COL1 and COL2, respectively. Tracking was performed for the Gaussian beam with $\sigma=3.3 \mathrm{ps}$ core in the presence of the longitudinal bunch back and forward tails. All the steering coils are turned on, with the horizontal offset of $2.6 \mathrm{~mm}$ at the second cavity and the common vertical offset of $2 \mathrm{~mm}$ at all three cavities. 
TABLE VIII. Population of particles at the core and the halo in the measurements and the simulations.

\begin{tabular}{lccccc}
\hline \hline & \multicolumn{2}{c}{ Measurement } & & \multicolumn{2}{c}{ Simulation } \\
\cline { 2 - 3 } \cline { 5 - 6 } Place of observation & Core, \% & Halo, \% & & Core, \% & Halo, \% \\
\hline SCM8 & 99.45 & 0.55 & & 99.07 & 0.93 \\
SCM16 & 99.37 & 0.63 & & 99.43 & 0.57 \\
SCM17 & 99.64 & 0.36 & & 99.50 & 0.50 \\
SCM21A & 99.48 & 0.52 & & 99.48 & 0.52 \\
Average & 99.49 & 0.51 & & 99.37 & 0.63 \\
\hline \hline
\end{tabular}

the simulated profiles)] of the profile. Then one can find a core-halo ratio, concerning the halo part to be less than $1 \%$ of the core intensity. The results of this procedure are summarized in Table VIII. Good agreements are obtained between the measurements and the simulations. It once again demonstrates the validity of this study.

Another important question is how much the spatial halo impacts the overall beam loss. To answer this question, more simulation studies should be performed. However, we have no exact beam loss measurement data to compare. All we have is the estimation of particle losses from the measurement of the secondarily produced photons during the high average current operation (the average beam current is $J=0.95 \mathrm{~mA}$ ) [24] at the concrete shields on the roof.

In this measurement, the dose rates on the cERL concrete roof of the thickness $100 \mathrm{~cm}$ (which is the thinnest shield) were measured by a $\mathrm{NaI}(\mathrm{Tl})$ scintillation survey meter along the beam line after one day of high-current operation [17]. The strong activation corresponds to the contact dose rate of $14 \mu \mathrm{Sv} / h$ at the copper beam collimator. And the weak activation corresponds to the contact dose rate of $0.04-0.9 \mu \mathrm{Sv} / \mathrm{h}$ at the stainless steel beam ducts. Then, the dose rate was calculated using the MARS15 code. For example, for a $20 \mathrm{MeV}$ beam and a loss rate of $1 \mathrm{nA}$, the calculation yielded a dose rate of $0.027 \mu \mathrm{Sv} / \mathrm{h}$. Finally, beam loss rates were evaluated by comparison between the measured and the calculated dose rates. Evaluated beam loss rates are indicated in blue (in percentage of the bunch population) in Fig. 24 at the corresponding beam line locations.

To simulate beam loss rates we change some input parameters from the previous ones. Mostly they are identical to those in Table IV, except the number of particles $N=10^{6}$, the beam current $J=0.95 \mathrm{~mA}$ (the same with the measurement described above), and the corresponding bunch charge $Q_{\mathrm{tot}}=J / f_{R F}=0.73 \mathrm{pC}$. Collimators COL1, 2 , and 4 inserted in as in the beam loss measurement. Effects of the injector steering coils were also included in the beam loss simulation. The measured horizontal offset of the injector middle cavity $(2.6 \mathrm{~mm})$ is included, as well as the common vertical offset of the three injector cavities $(2 \mathrm{~mm})$ evaluated in the previous section. Then the beam distribution with 3.3 ps Gaussian core with the back and forward tails was tracked. The simulated beam loss rates are indicated by italic red numbers in Fig. 24. Remarkably, the measured (not directly but evaluated through the procedures) and the simulated loss rates are comparable in the early part of the ring (the collimator COL4). It seems that toward the end of the ring, other unknown beam loss factors (not investigated in this work) come in and the measured loss rates surpass the simulated ones. The total beam loss in the recirculation loop is $0.027 \%$ in percentage of the bunch population according to the experimental evaluation, while it is $0.0081 \%$ in the simulations.

In the process of understanding the simulated beam loss distribution, we found that there seems to be several driving mechanisms for beam loss. One is the large betatron functions at several locations in the north straight section

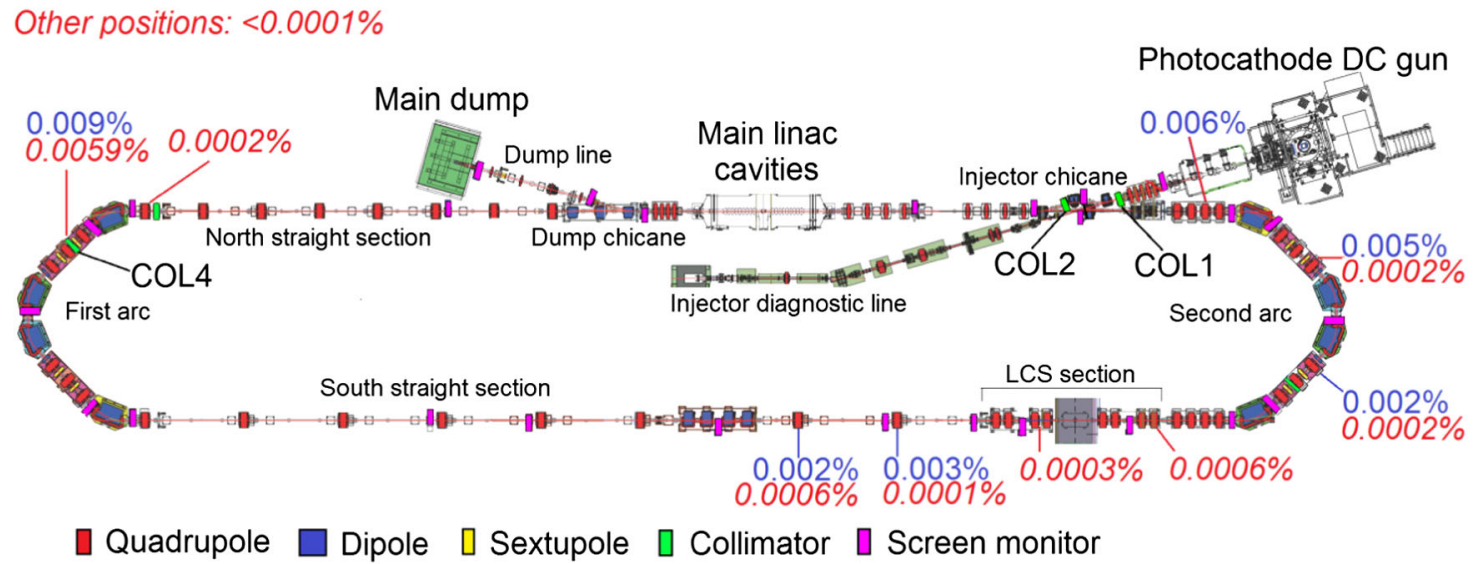

FIG. 24. Comparison of beam loss rates along the beam line for the high-current operation $(J=0.95 \mathrm{~mA})$ where the collimators COL1, 2 and 4 are inserted in. Loss rates are given in percentage of the bunch population. Loss rates estimated from the measured secondary photons are denoted by blue numbers. Simulation results (italic red numbers) are for particles tracking with the total number of particles $N=10^{6}$, with the Gaussian distribution of the $\sigma=3.3 \mathrm{ps}$ core in the presence of longitudinal bunch back and forward tails. In the simulations, all the steering coils are turned on. The measured horizontal offset of the second cavity $(2.6 \mathrm{~mm})$, and the common vertical offsets of the cavities nos. 1-3 (2 mm) are included. 
(the upper half in Fig. 24) as well as in the south straight section at the LCS section location. See Fig. 2 for more details. Another significant loss enhancement factor is the collimator COL4 located at the first arc. The losses at the arc sections occur mostly due to the nonzero dispersion.

\section{CONCLUSIONS}

Non-negligible transverse halos have been experimentally observed at the cERL. The present study is aimed at finding the main causes of the beam halo at the cERL and explaining its formation mechanisms. It seems that these halo formation mechanisms generate transverse offsets of a beam at the collimators COL1 and COL2 in the injector line since they are quite effective in cutting the beam halo. The simulated beam losses show the reasonable agreements with the measured values for both the loss rates and the beam loss distribution along the beam line.

Based on the experimental data, we made a conjecture that the most likely cause of the beam halo at the cERL is the longitudinal bunch tail originated at the photocathode, and its transfer to a vertical halo in the rest of the machine. In this transfer mechanism, the injector cavities and the displacement of the beam orbit inside them are essential elements. The accelerating mode of the injector cavities can produce transverse rf kicks to nonrelativistic particles when the beam orbit has a transverse offset. The strength (and even the direction in some cases) of those kicks is different for particles in the core and those in the tails, and thus some particles in the tail start to deviate transversely from the core, resulting in a halo. We found that the steering coils (and possibly the ambient magnetic fields) produce substantial beam displacement in the injector cavities. The present halo simulations also show a possibility that all three injector cavities are shifted up together by $2 \mathrm{~mm}$ due to a vertical shift of the entire cryomodule. These beam trajectory displacements inside the injector cavities are found to be a significant halo formation mechanism at the cERL. It may be the first time to prove that the transverse halo can be formed from the longitudinal bunch tail by combination of the $\mathrm{rf}$ accelerating (mono-pole) fields of cavities and the transverse offset of nonrelativistic particles in the "semilinear" system. We must emphasize that this mechanism is a quite new finding and different from other observed similar phenomena in which transverse halos are believed to be generated from longitudinal tails due to other mechanisms, mostly by nonlinear effects such as the space charge.

We found that the inclusion of the forward tail in simulations significantly improves agreements between experimental data and simulation results. It is a tiny tail outstripping the core of the beam, though its origin is not well understood. It could occur if the laser pulse profile slightly differs from the Gaussian one or the stray light from the laser system arrives slightly earlier at the cathode than the main laser pulse.
The transformation of a longitudinal tail to a transverse halo provides an interesting mechanism in which even the linear system can generate a significant beam halo without nonlinear effects such as the space charge force or CSR. One possible cure for this halo will be realignment of the cryomodule so that the beam goes through its center. Another possible cure is to reduce the bunch tails by changing the photocathode material from the present GaAs to a multi-alkali that is known to have a shorter longitudinal tail [58]. Multi-alkali photocathodes for the cERL have been developed and will be available soon [59-60]. We will try one or both of these cures in the near future. Once these cures are applied, we believe that the present beam halo will be mitigated significantly. Then, the next step of the cERL $\mathrm{R} \& \mathrm{D}$ will be the operation with the low-emittance and the high bunch charge (or high average beam current) [61]. For this end, more operational studies on the mechanisms of the beam halo formation are required to achieve overall beam loss reduction. The space charge effect will be another important factor in a higher bunch charge operation. They should be subjects of the further study.

\section{ACKNOWLEDGMENTS}

The authors would like to thank the cERL commissioning team and the operation staff for their cooperation and help during the machine study. We also would like to acknowledge Professor Y. Kobayashi for his comprehensive assistance and Professor S. Sakanaka for the overall consultations. Dr. Y. Honda should be acknowledged for his contributions concerning the laser system of the cERL. A special thank should be given to Dr. M. Yamamoto for multiple photocathode-related discussions. The research leading to these results has received funding from Grant-inAid for Creative Scientific Research of JSPS (KAKENHI 15K04747).

\section{APPENDIX A: DATA FITTING ALGORITHM}

The data fitting algorithm is based on the nonlinear least squares method. Its goal is to adjust the $m$ parameters that are held in vector $\mathbf{p}=\left(p_{1}, p_{2}, \ldots, p_{j}\right), j=\overline{1, m}$ of a model function $f\left(x_{i}, \mathbf{p}\right)$ for the best fit of data set $\left(x_{i}, y_{i}\right), i=\overline{1, n}$, where $x_{i}$ is an independent variable and $y_{i}$ is a dependent variable [62]. In order to perform a nonlinear least squares curve fit, one needs to minimize the sum $S$ of squares of the residuals $r_{i}$ :

$$
\begin{gathered}
r_{i}=y_{i}-f\left(x_{i}, \mathbf{p}\right), \\
S=\sum_{i=1}^{n} r_{i}^{2} .
\end{gathered}
$$

Then, we use the minimization routine based on the NelderMead simplex algorithm. Following Lagarias et al. in [63], the algorithm parameters are determined as (i) reflection coefficient $\alpha>0$ (typical value is $\alpha=1$ ); (ii) shrinking 
coefficient $\beta>0$ (typical value is $\beta=0.5$ ); and (iii) expansion coefficient $\gamma>0$ (typical value is $\gamma=2$ ). (1) Preparation.-Initially $n+1$ points of $z_{i}=\left(z_{i}^{(1)}\right.$, $\left.z_{i}^{(2)}, \ldots, z_{i}^{(n)}\right), i=\overline{1, n+1}$ are selected to form a simplex of $n$-dimensional space. At these points, the function values are calculated as $z_{1}=z\left(x_{1}\right), z_{2}=z\left(x_{2}\right), \ldots, z_{n+1}=$ $z\left(x_{n+1}\right)$. (2) Sorting.-Select three points from the simplex vertices: $x_{h}$ with the largest (of the selected) value of the function $z_{h} ; x_{g}$ with the next largest value $z_{g} ; x_{l}$ and with the least value of the function $z_{l}$. The aim of the following manipulations is at least $z_{h}$ reduction. (3) Find the center of gravity of all the points, except $x_{h}: x_{c}=1 / n \sum_{i \neq h} x_{i}$. (4) Reflection.-Reflect a point $x_{h}$ relative to $x_{c}$ with coefficient $\alpha$ (if $\alpha=1$, it is the central symmetry; in general it is homothety). Thus we find the point $x_{r}$ and calculate the function at this point: $z_{r}=z\left(x_{r}\right)$. The coordinates of the new point are calculated by the formula $x_{r}=(1+\alpha) x_{c}-\alpha x_{h}$. (5) Look at how we have managed to reduce the function look for a place of $z_{r}$ in the $z_{h}, z_{g}, z_{l}$ series. First, if $z_{r}<z_{l}$, then we succeed, and we can try to increase the step and perform "expansion." A new point is $x_{e}=(1-\gamma) x_{c}+\gamma x_{r}$, and the function value is $z_{e}=z\left(x_{e}\right)$. Second, if $z_{e}<z_{r}$, then it is possible to expand the simplex up to this point. Assign the value of $x_{e}$ to the point $x_{h}$, and go to step 9. Third, if $z_{r}<z_{e}$, then we moved too far. Assign the value of $x_{r}$ to the point $x_{h}$, and go to step 9. Fourth, if $z_{l}<z_{r}<z_{g}$, then the choice of the point is not bad (the new one is better than the older two). Assign the value of $x_{r}$ to the point $x_{h}$, and go to step 9. Fifth, if $z_{g}<z_{r}<z_{h}$, then swap $x_{r}$ and $x_{h}$ values. There is also a need to swap $z_{r}$ and $z_{h}$ values. After that, go to step 6 . Finally, if $z_{h}<z_{r}$, then go to the next step 6 .

As a result (possibly after renumbering) we get $z_{l}<z_{g}<z_{h}<z_{r}$. (6) Shrinking.-Construct a point $x_{s}=(1-\beta) x_{c}+\beta x_{h}$, and calculate the value $z_{s}=z\left(x_{s}\right)$. (7) If $z_{s}<z_{h}$, then assign the value of $x_{s}$ the point $x_{h}$, and go to step 9. (8) If $z_{s}<z_{h}$, then the initial points were the most successful. Make a "global shrinking" of the simplex called homothety at the point with the least value $x_{l}$ : $x_{i} \leftarrow x_{l}+\left(x_{i}-x_{l}\right) / 2, i \neq l$.

The last step is a convergence examination. It may be performed in different ways, for example, by estimation of the variance of the points set. The essence of the examination is to check the mutual proximity of obtained vertices of the simplex, which implies that their proximity is to the required minimum. If the required accuracy is not reached, one can continue with the iteration from step 2 .

\section{APPENDIX B: ANAYLTICAL EVALUATION OF THE CONVOLUTION INTEGRAL OF NORMAL DISTRIBUTION WITH THE PHOTOEMISSION CURRENT FUNCTION}

According to Eqs. (1)-(3), the functions to be convoluted have the following forms:

$$
\begin{gathered}
I(k)=\frac{1}{\sqrt{\pi k}}-e^{k} \operatorname{erfc}(\sqrt{k}), \\
G(t)=\frac{1}{\sqrt{2 \pi} \sigma} e^{-\frac{t^{2}}{2 \sigma^{2}}},
\end{gathered}
$$

where $k=t / \tau$. Therefore

$$
I(t)=\sqrt{\frac{\tau}{\pi t}}-e^{\frac{t}{\tau}} \operatorname{erfc}\left(\sqrt{\frac{t}{\tau}}\right) .
$$

And the convolution integral becomes

$$
\begin{aligned}
(I * G)(t) & =\int_{0}^{\infty} I(y) G(t-y) d y \\
& =\frac{1}{\sqrt{2 \pi} \sigma} \int_{0}^{\infty}\left[\sqrt{\frac{\tau}{\pi y}}-e^{\frac{y}{\tau}} \operatorname{erfc}\left(\sqrt{\frac{y}{\tau}}\right)\right] e^{-\frac{(t-y)^{2}}{2 \sigma^{2}}} d y .
\end{aligned}
$$

Since $\operatorname{erfc}(z)=1-\operatorname{erf}(z)$ [see Eq. (4)] we have

$$
\begin{aligned}
(I * G)(t)= & \frac{\sqrt{\tau}}{\sqrt{2} \pi \sigma} \underbrace{\int_{0}^{\infty} \frac{1}{\sqrt{y}} e^{-\frac{(t-y)^{2}}{2 \sigma^{2}}} d y}_{I_{1}} \\
& -\frac{1}{\sqrt{2 \pi} \sigma} \underbrace{\int_{0}^{\infty} e^{\frac{y}{\tau}} e^{-\frac{(t-y)^{2}}{2 \sigma^{2}}} d y}_{I_{2}} \\
& +\frac{1}{\sqrt{2 \pi} \sigma} \underbrace{\int_{0}^{\infty} e^{\frac{y}{\tau}} \operatorname{erf}\left(\sqrt{\frac{y}{\tau}}\right) e^{-\frac{(t-y)^{2}}{2 \sigma^{2}}} d y}_{I_{3}} .
\end{aligned}
$$

Let us consider the integrals $I_{1}, I_{2}$, and $I_{3}$ separately in what follows. Let us work out $I_{1}$ first:

$I_{1}=\int_{0}^{\infty} \frac{1}{\sqrt{y}} e^{-\frac{(t-y)^{2}}{2 \sigma^{2}}} d y=e^{-\frac{t^{2}}{2 \sigma^{2}}} \int_{0}^{\infty} \frac{1}{\sqrt{y}} e^{-\frac{y^{2}}{2 \sigma^{2}}+\frac{t y}{\sigma^{2}}} d y$.

We apply the following relation [64] to Eq. (B6):

$$
\int_{0}^{\infty} x^{\alpha-1} e^{-p x^{2}-q x} d x=\Gamma(\alpha)(2 p)^{-\frac{\alpha}{2}} e^{\frac{q^{2}}{8 p}} D_{-\alpha}\left(\frac{q}{\sqrt{2 p}}\right) .
$$

Here $\operatorname{Re}(\alpha)>0$ and $\operatorname{Re}(p)>0, D_{\nu}(z)$ is the parabolic cylinder function

$$
D_{\nu}(z)=2^{\frac{\nu}{2}} e^{-\frac{z^{2}}{4}} \Psi\left(-\frac{\nu}{2} ; \frac{1}{2} ; \frac{z^{2}}{2}\right)
$$

and 


$$
\begin{aligned}
\Psi(a ; c ; z)= & \Gamma\left[\begin{array}{c}
1-c \\
1+a-c
\end{array}\right]{ }_{1} F_{1}(a ; c ; z) \\
& +\Gamma\left[\begin{array}{c}
c-1 \\
a
\end{array}\right] z^{1-c}{ }_{1} F_{1}(1+a-c ; 2-c ; z)
\end{aligned}
$$

is the confluent hypergeometric function [64]. For $\alpha=1 / 2$, $p=1 / 2 \sigma^{2}$, and $q=-t / \sigma^{2}$ one can get

$$
I_{1}=\frac{\sqrt{\pi \sigma}}{2^{\frac{1}{4}}} e^{-\frac{t^{2}}{2 \sigma^{2}} \Psi}\left(\frac{1}{4} ; \frac{1}{2} ; \frac{t^{2}}{2 \sigma^{2}}\right)
$$

Note that $\Gamma(1 / 2)=\sqrt{\pi}$. Next, we move to the integral $I_{2}$ :

$I_{2}=\int_{0}^{\infty} e^{\frac{y}{\tau}} e^{-\frac{(t-y)^{2}}{2 \sigma^{2}}} d y=e^{-\frac{t^{2}}{2 \sigma^{2}}} \int_{0}^{\infty} e^{-\frac{y^{2}}{2 \sigma^{2}}-\left(-\frac{t}{\sigma^{2}}-\frac{1}{\tau}\right) y} d y$.

Using the relation [64]

$$
\int_{0}^{\infty} e^{-p x^{2}-q x}=\frac{1}{2} \sqrt{\frac{\pi}{p}} e^{\frac{q^{2}}{4 p}} \operatorname{erfc}\left(\frac{q}{2 \sqrt{p}}\right), \quad \operatorname{Re}(p)>0,
$$

we obtain for $p=1 / 2 \sigma^{2}$ and $q=-t / \sigma^{2}-1 / \tau$

$$
I_{2}=\sigma \sqrt{\frac{\pi}{2}} e^{\frac{\sigma^{2}}{2 \tau^{2}}+\frac{t}{\tau}} \operatorname{erfc}\left(-\frac{t \tau+\sigma^{2}}{\sqrt{2} \sigma \tau}\right)
$$

The integral $I_{3}$ can be expanded as

$$
\begin{aligned}
I_{3} & =\int_{0}^{\infty} e^{\frac{y}{\tau}} \operatorname{erf}\left(\sqrt{\frac{y}{\tau}}\right) e^{-\frac{(t-y)^{2}}{2 \sigma^{2}}} d y \\
& =\frac{2}{\sqrt{\pi}} \sum_{n=0}^{\infty} \frac{(-1)^{n}}{\tau^{n+1 / 2} n !(2 n+1)} e^{-\frac{t^{2}}{2 \sigma^{2}}} \int_{0}^{\infty} y^{n+\frac{1}{2}} e^{-\frac{y^{2}}{2 \sigma^{2}}\left(-\frac{t}{\sigma^{2}}-\frac{1}{\tau}\right) y} d y .
\end{aligned}
$$

Then we apply Eq. (B7) again, with $\alpha=n+3 / 2$, $p=1 / 2 \sigma^{2}$, and $q=-t / \sigma^{2}-1 / \tau$. This yields

$$
\begin{aligned}
I_{3}= & \frac{2^{\frac{1}{4}} \sigma^{\frac{3}{2}}}{\sqrt{\pi \tau}} e^{-\frac{t^{2}}{2 \sigma^{2}}} \sum_{n=0}^{\infty} \frac{(-1)^{n} \sigma^{n} \Gamma\left(n+\frac{3}{2}\right)}{\tau^{n} 2^{\frac{n}{2}} n !(2 n+1)} \Psi \\
& \times\left(\frac{n}{2}+\frac{3}{4} ; \frac{1}{2} ; \frac{\left(t \tau+\sigma^{2}\right)^{2}}{2 \sigma^{2} \tau^{2}}\right) .
\end{aligned}
$$

Applying the following properties of the Gamma function [64]

$$
\begin{aligned}
\Gamma\left(n+\frac{1}{2}\right) & =\frac{\sqrt{\pi}}{2^{n}}(2 n-1) ! !, \text { therefore, } \\
\Gamma\left(n+\frac{3}{2}\right) & =\frac{\sqrt{\pi}}{2^{n+1}}(2 n+1) ! !,
\end{aligned}
$$

one can derive the final form:

$$
\begin{aligned}
I_{3}= & \frac{\sigma^{\frac{3}{2}}}{2^{\frac{3}{4}}} \frac{1}{\sqrt{\tau}} e^{-\frac{t^{2}}{2 \sigma^{2}}} \sum_{n=0}^{\infty} \frac{(-1)^{n} \sigma^{n}(2 n-1) ! !}{\tau^{n} 2^{\frac{3 n}{2}} n !} \Psi \\
& \times\left(\frac{n}{2}+\frac{3}{4} ; \frac{1}{2} ; \frac{\left(t \tau+\sigma^{2}\right)^{2}}{2 \sigma^{2} \tau^{2}}\right) .
\end{aligned}
$$

By combining all these results, we get the final convolution formula:

$$
\begin{aligned}
(I * G)(t)= & \frac{\sqrt{\tau}}{2^{\frac{3}{4}} \sqrt{\pi \sigma}} e^{-\frac{t^{2}}{2 \sigma^{2}} \Psi}\left(\frac{1}{4} ; \frac{1}{2} ; \frac{t^{2}}{2 \sigma^{2}}\right) \\
& -\frac{1}{2} e^{\frac{\sigma^{2}}{2 \tau^{2}}+\frac{t}{\tau}} \operatorname{erfc}\left(-\frac{t \tau+\sigma^{2}}{\sqrt{2} \sigma \tau}\right) \\
& +\frac{\sigma}{2^{\frac{5}{4}}} \frac{1}{\sqrt{\pi \tau}} e^{-\frac{t^{2}}{2 \sigma^{2}}} \sum_{n=0}^{\infty} \frac{(-1)^{n} \sigma^{n}(2 n-1) ! !}{\tau^{n} 2^{\frac{3 n}{2}} n !} \Psi \\
& \times\left(\frac{n}{2}+\frac{3}{4} ; \frac{1}{2} ; \frac{\left(t \tau+\sigma^{2}\right)^{2}}{2 \sigma^{2} \tau^{2}}\right) .
\end{aligned}
$$

\section{APPENDIX C: MAGNETIC FIELDS OF A PAIR OF RECTANGULAR LOOPS}

The vector components of magnetic flux density are evaluated for the rectangular loop (see Fig. 25). A single loop with a small cross section carrying a current $I$ is placed on the $y=0$ plane. The origin of the coordinate system is located at the center of the loop. The loop has dimensions of $2 a$ (for the $x$ direction) and $2 b$ (for the $y$ direction), respectively. The vector $\mathbf{R}$ is a radius vector of the point of observation $P(x, y, z)$, where the magnetic flux density will

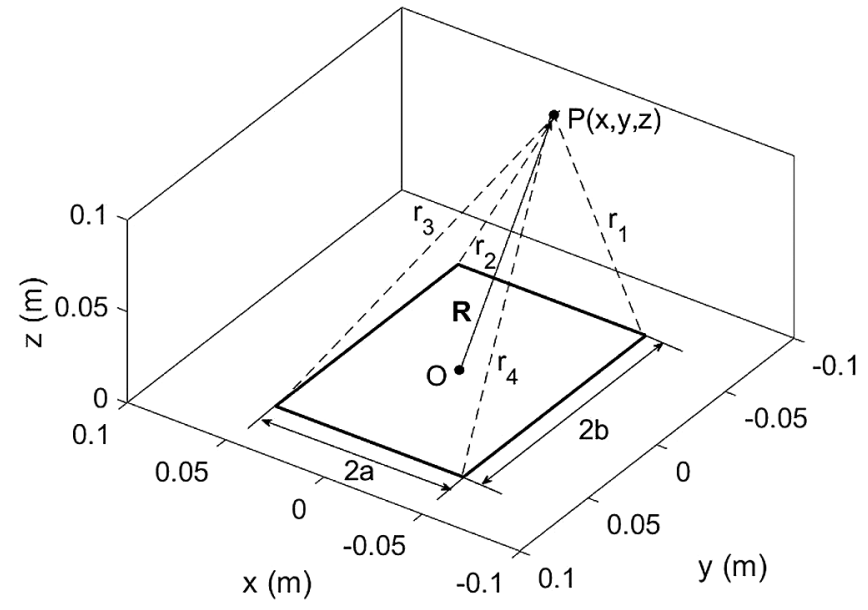

FIG. 25. Geometry of a single rectangular loop. 
be obtained. The parameters $r_{1}, r_{2}, r_{3}$, and $r_{4}$ are the distances from the point of observation to the corners of the loop. Let us evaluate the vector components of magnetic flux density using the vector potential.

The expressions for the vector potentials $A_{x}$ and $A_{z}$ on the $y=0$ plane as well as its relations with the magnetic flux density components $B_{x}, B_{y}$, and $B_{z}$ are well known (see, for example, [65]):

$$
\begin{aligned}
& A_{x}=\frac{\mu_{0} I}{4 \pi} \ln \left[\frac{\left(r_{1}+a+x\right)\left(r_{3}-a+x\right)}{\left(r_{2}-a+x\right)\left(r_{4}+a+x\right)}\right], \\
& A_{z}=\frac{\mu_{0} I}{4 \pi} \ln \left[\frac{\left(r_{2}+b+z\right)\left(r_{4}-b+z\right)}{\left(r_{3}-b+z\right)\left(r_{1}+b+z\right)}\right] .
\end{aligned}
$$

and

$$
B_{x}=-\frac{\partial A_{z}}{\partial y}, \quad B_{y}=\frac{\partial A_{z}}{\partial x}-\frac{\partial A_{x}}{\partial z}, \quad B_{z}=\frac{\partial A_{x}}{\partial y} .
$$

Here $\mu_{0}=4 \pi \cdot 10^{-7} N / A^{2}$ is the permeability of free space.

By taking a curl of $\mathbf{A}$ in Cartesian coordinates, one can obtain:

$B_{x}=\frac{\mu_{0} I}{4 \pi} \sum_{k=1}^{4} \frac{(-1)^{k+1} y}{r_{k}\left(r_{k}+d_{k}\right)}, \quad B_{z}=\frac{\mu_{0} I}{4 \pi} \sum_{k=1}^{4} \frac{(-1)^{k+1} y}{r_{k}\left(r_{k}+c_{k}\right)}$,

and

$B_{y}=\frac{\mu_{0} I}{4 \pi} \sum_{k=1}^{4}(-1)^{k}\left(\frac{c_{k}}{r_{k}\left(r_{k}+d_{k}\right)}+\frac{d_{k}}{r_{k}\left(r_{k}+c_{k}\right)}\right)$,

where

$$
\begin{aligned}
& c_{1}=x+a, \quad d_{1}=z+b, \\
& r_{1}=\sqrt{(x+a)^{2}+(z+b)^{2}+y^{2},} \\
& c_{2}=x-a, \quad d_{2}=z+b, \\
& r_{2}=\sqrt{(x-a)^{2}+(z+b)^{2}+y^{2},} \\
& c_{3}=x-a, \quad d_{3}=z-b, \\
& r_{3}=\sqrt{(x-a)^{2}+(z-b)^{2}+y^{2},} \\
& c_{4}=x+a, \quad d_{4}=z-b, \\
& r_{4}=\sqrt{(x+a)^{2}+(z-b)^{2}+y^{2}} .
\end{aligned}
$$

For the system of two rectangular loops (see Fig. 26), the explicit expressions of $B_{x}, B_{y}$, and $B_{z}$ can be easily written down using the superposition theorem. Thus, $y$ should be

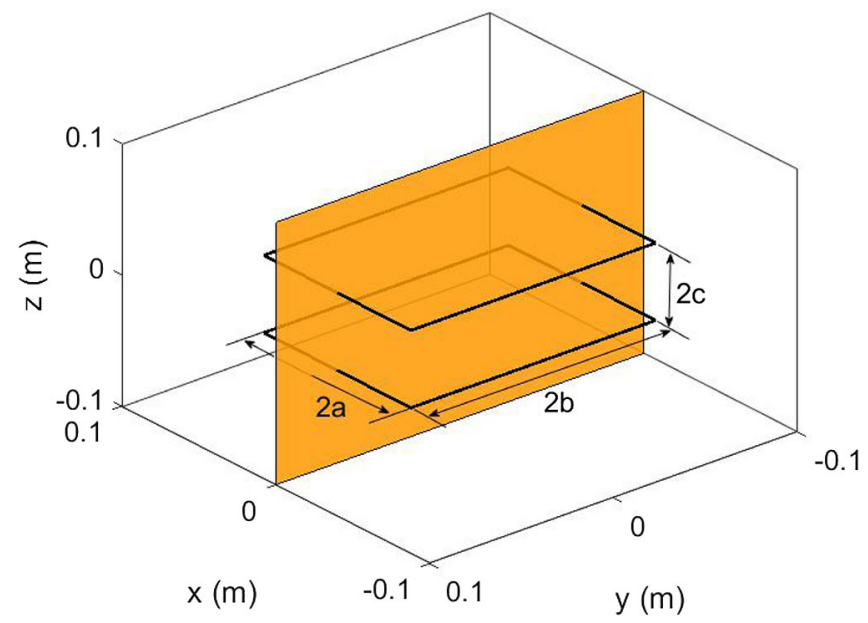

FIG. 26. Geometry of a pair of rectangular loops and the $z-y$ plane.

replaced by $y-y_{i}$, where $y_{i}(i=1,2)$ is the position of the $i$ loop.

The magnetic field lines on the $z-y$ plane are given by the contours of the scalar function $A_{x}(0, y, z)$ since $\mathbf{B}$. $\nabla A_{x}(0, y, z)=0$ and they are shown in Fig. 27. Figures 28 and 29 show $B_{y}$ as a function of $z$ and $B_{y}$ as a function of $y$, respectively. Note that the magnetic field $B_{y}$ is uniform in the region between the two loops as shown in Fig. 28.

The magnetic field components can be also calculated using Bio-Savart law [56] by summing fields produced by the four segments of the current loop (Fig. 25):

$$
\mathbf{B}=\int_{\text {wire }} d \mathbf{B}=\frac{\mu_{0} I}{4 \pi} \int_{\text {wire }} \frac{d \mathbf{l} \times \mathbf{R}}{R^{2}},
$$

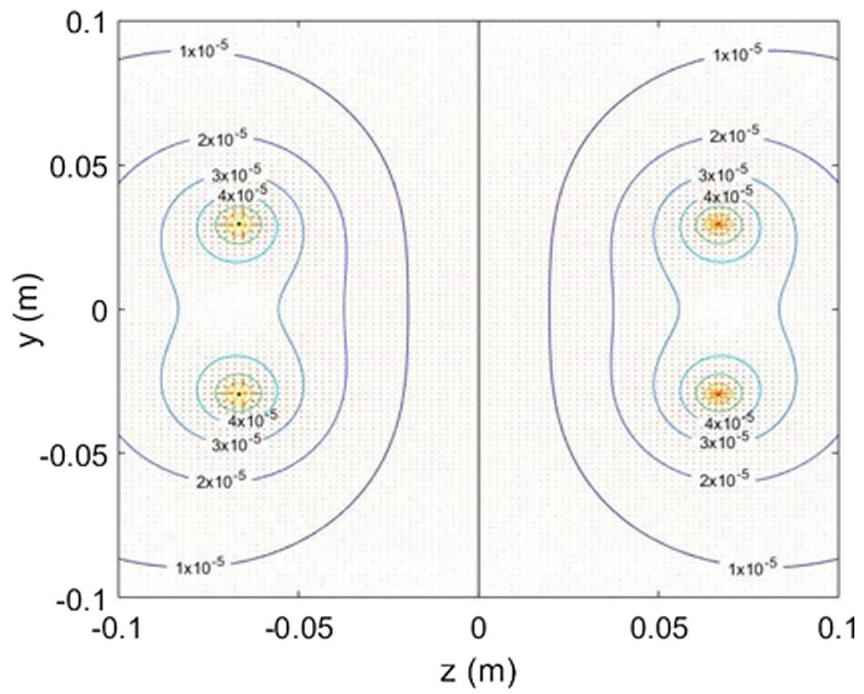

FIG. 27. $B_{y}$ component of the magnetic flux density on the $z-y$ plane. 


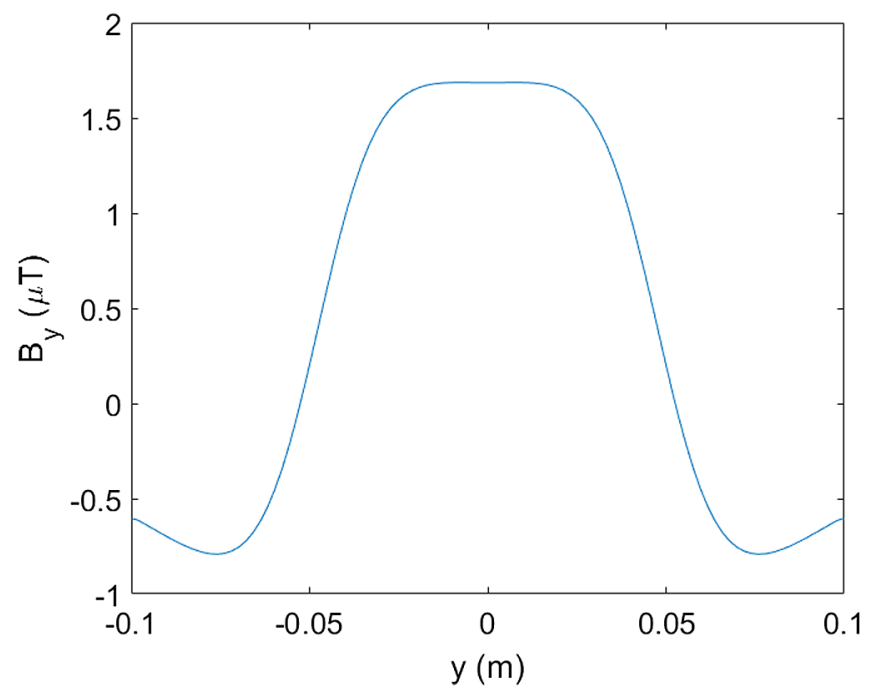

FIG. 28. $\quad B_{y}$ component along the longitudinal coordinate $z$.

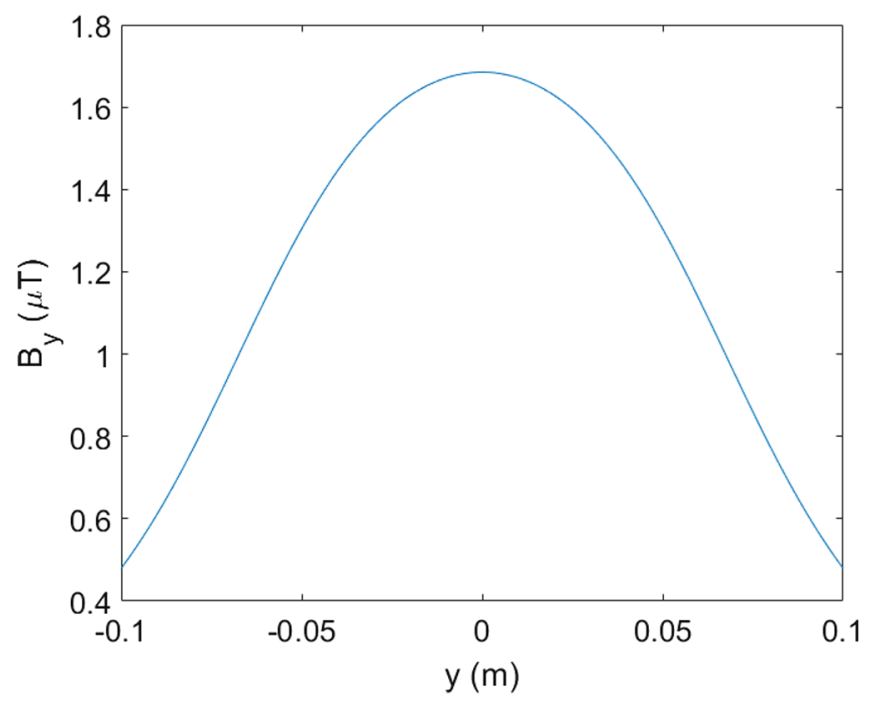

FIG. 29. $B_{y}$ component along the transverse coordinate $y$.

where $I \cdot d \mathbf{l}$ is the current-carrying element. Thus, the field components can be written as

$$
\begin{aligned}
d B_{x} & =\frac{I}{4 \pi R^{3}}\left(d l_{z} R_{y}\right), \\
d B_{y} & =\frac{I}{4 \pi R^{3}}\left(d l_{x} R_{z}-d l_{z} R_{x}\right), \\
d B_{z} & =\frac{I}{4 \pi R^{3}}\left(-d l_{x} R_{y}\right) .
\end{aligned}
$$

The $B_{x}$ component includes fields produced by the segments parallel to the $z$ axis, and the $B_{z}$ component includes fields produced by the segments parallel to the $x$ axis. The $B_{y}$ component includes fields produced by all the segments of the loop. Their forms in Eq. (C7) are probably more convenient for numerical evaluations and could be useful for computer programming.
[1] V. Fedotov, Mechanisms of halo formation, AIP Conf. Proc. 693, 3 (2003).

[2] K. Wittenberg, in Beam Diagnostics, Proceedings of CERN Accelerator School, Dourdan, 2008, edited by D. Brandt (CERN, Dourdan, 2009), p. 557, https://cds.cern .ch/record/1213291/files/p557.pdf.

[3] T. Tenenbaum, P. Emma, L. Keller, Y. Nosochekov, T. Raubenheimer, and M. Woodly, in Proceedings of 2001 Particle Accelerator Conference, Chicago, 2001 (IEEE, Chicago, 2001), p. 3840.

[4] L. Merminga, J. Irwin, R. Helm, and R. D. Ruth, SLACPUB-5436, 1991.

[5] J.M. Lagniel, On halo formation from space-charge dominated beams, Nucl. Instrum. Methods Phys. Res., Sect. A 345, 46 (1994).

[6] A. Piwinski, DESY Report № 98-179, 1998, https://arxiv .org/abs/physics/9903034v1.

[7] S. Khan, in Proceedings of the 4th European Particle Accelerator Conference, London, 1994, edited by V.P. Suller and C. Petit-Jean-Genaz (World Scientific Publishing, Singapore, 1995), p. 1192.

[8] G. Hoffstaetter, M. P. Ehrlichman, and A. Temnykh, in Proceedings of the 11th European Particle Accelerator Conference, Genoa, 2008 (EPS-AG, Genoa, 2008), p. 1631, http://accelconf.web.cern.ch/AccelConf/e08/ papers/tupp040.pdf.

[9] V. Fedotov, in Proceedings of the 50th ICFA Advanced Beam Dynamics Workshop, Tsukuba, 2011, edited by A. Shirakawa (KEK, Tsukuba, 2011), p. 73, http://accelconf .web.cern.ch/AccelConf/ERL2011/papers/proceed.pdf.

[10] V. Fedotov, Beam halo formation in high-intensity beams, Nucl. Instrum. Methods Phys. Res., Sect. A 557, 216 (2006).

[11] C. Bocchetta, in Synchrotron Radiation and Free-Electron Lasers, proceedings of CERN Accelerator School, Grenoble, 1996, edited by S. Turner (CERN, Geneva, 1998) p. 221, https://cds.cern.ch/record/362891/files/CERN-98-04.pdf.

[12] H. Abe, S. Adachi, K. Amemiya, T. Arima, K. Asakura, L. Chavas, N. Funamori, T. Furuya, R. Hajima, and K. Harada et al., KEK Report No. 2012-4, 2012, http://pfwww.kek.jp/ ERLoffice/detabase/ERL_CDR_full_text.pdf.

[13] R. Hajima, N. Nakamura, S. Sakanaka, and Y. Kobayashi, KEK Report № 2007-7, JAEA-Research Report No. 2008032, 2008 (in Japanese), http://jolissrch-inter.tokai-sc.jaea .go.jp/pdfdata/JAEA-Research-2008-032.pdf.

[14] S. Sakanaka, M. Akemoto, T. Aoto, D. A. Arakawa, S. Asaoka, A. Enomoto, S. Fukuda, K. Furukawa, T. Furuya, $\mathrm{K}$. Haga et al., in Proceedings of the 1st International Particle Accelerator Conference, Kyoto, 2010, edited by A. Noda, C. Petit-Jean-Genaz, V. Schaa, T. Shirai, and A. Shirakawa (JACoW, Geneva, 2010), p. 2338, http://epaper .kek.jp/IPAC10/papers/tupe091.pdf.

[15] O. Tanaka, N. Nakamura, M. Shimada, T. Miyajima, and S. Sakanaka, in Proceedings of the 6th International Particle Accelerator Conference, Richmond, 2015, edited by S. Henderson, T. Satogata, and V. R. W. Schaa (JACoW, Geneva, 2015), p. 1587, https://jacowfs.jlab.org/conf/ proceedings/IPAC2015/papers/tupwa068.pdf.

[16] O. A. Konstantinova, N. Nakamura, M. Shimada, K. Harada, K. Umemori, H. Sakai, T. Furuya, and E. Cenni, in Proceedings of the 5th International Particle 
Accelerator Conference, Dresden, 2014, edited by C. PetitJean-Genaz, G. Arduini, P. Michel, and V. R. W. Schaa (JACoW, Geneva, 2014), p. 349, http://accelconf.web.cern .ch/AccelConf/IPAC2014/papers/mopro109.pdf.

[17] S. Sakanaka, K. Haga, Y. Honda, H. Matsumura, T. Miyajima, T. Nogami, T. Obina, H. Sagehashi, M. Shimada, and M. Yamamoto, in Proceedings of the 7th International Particle Accelerator Conference, Busan, 2016, edited by C. Petit-Jean-Genaz, D. E. Kim, K. S. Kim, I. S. Ko, K. R. Kim, and V. R. W. Schaa (JACoW, Geneva, 2016), p. 1839, http://accelconf.web.cern.ch/ AccelConf/ipac2016/papers/tupow038.pdf.

[18] I. Bazarov, B. Dunham, Y. Li, X. Liu, D. Ouzounov, C. Sinclair, F. Hannon, and T. Miyajima, Thermal emittance and response time measurements of negative electron affinity photocathodes, J. Appl. Phys. 103, 054901 (2008).

[19] K. Aulenbacher, J. Schuler, D. V. Harrach, E. Reichert, J. Röthgen, A. Subashev, V. Tioukine, and Y. Yashin, Pulse response of thin III/V semiconductor photocathodes, J. Appl. Phys. 92, 7536 (2002).

[20] S. Benson et al., in Proceedings of 2007 Particle Accelerator Conference, Albuquerque, 2007 (IEEE, Albuquerque, 2007), p. 79.

[21] C. Tennant, in Proceedings of ERL17, Geneva, 2017 (CERN, Geneva, 2017), WEIBCC004_TALK, https://indico.cern.ch/ event/470407/contributions/2610923/attachments/1478707/ 2291920/WEIBCC004_TALK.PPTX.

[22] P. Evtushenko, in Proceedings of 33rd FEL Conference, Shanghai, 2011 (JACoW, Shanghai 2011), FROBl1, http:// epaper.kek.jp/FEL2011/talks/frobi1_talk.pdf.

[23] R. C. York and C. Reece, in Proceedings of 1987 IEEE Particle Accelerator Conference, Washington D.C., 1987 (IEEE, Washington D.C., 1987), p. 1307.

[24] H. Matsumura, K. Haga, K. Hozumi, T. Miura, S. Nagaguro, T. Obina, T. Oyama, S. Sakanaka, A. Toyoda, and N. Yoshihara, in Proceedings of the 7th International Particle Accelerator Conference, Busan, 2016, edited by C. Petit-Jean-Genaz, D. E. Kim, K. S. Kim, I. S. Ko, K. R. Kim, and V. R. W. Schaa (JACoW, Geneva, 2016), p. 2711, http://accelconf.web.cern.ch/AccelConf/ipac2016/papers/ wepor020.pdf.

[25] M. Shimada, in Proceedings of the 53th ICFA Advanced Beam Dynamics Workshop on Energy Recovery Linacs, Novosibirsk, 2013, edited by M. V. Kuzin and V. R. W. Schaa (BINP, Novosibirsk, 2013).

[26] R. Takai, T. Obina, H. Sagehashi, Y. Tanimoto, T. Honda, T. Nogami, and M. Tobiyama, in Proceedings of the 3rd International Beam Instrumentation Conference, Monterey, 2014, edited by J. Sebek, S. Smith, D. Butto, I. Costa, M. Montes-Loera, I. Martin, and V.R. W. Schaa (JACoW, Geneva, 2015), p. 7, http://accelconf.web.cern.ch/AccelConf/ IBIC2014/papers/mocyb2.pdf.

[27] P. Evtushenko, in Proceedings of 1st International Beam Instrumentation Conference, Tsukuba, 2012 (JACoW, Tsukuba, 2011), p. 263.

[28] Y. Tanimoto, S. Asaoka, T. Honda, T. Nogami, T. Obina, and R. Takai, in Proceedings of the 4th International Particle Accelerator Conference, Shanghai, 2013, edited by Z. Dai, C. Petit-Jean-Genaz, V. R. W. Schaa, and C. Zhang
(JACoW, Geneva, 2013), p. 3315, http://accelconf.web .cern.ch/AccelConf/IPAC2013/papers/thpfi012.pdf.

[29] N. Nishimori, R. Nagai, S. Matsuba, R. Hajima, M. Yamamoto, Y. Honda, T. Miyajima, H. Iijima, M. Kuriki, and M. Kuwahara, Experimental investigation of an optimum configuration for a high-voltage photoemission gun for operation at $\geq 500 \mathrm{kV}$, Phys. Rev. ST Accel. Beams 17, 053401 (2014).

[30] Y. Honda, S. Matsuba, X. Jin, T. Miyajima, M. Yamamoto, T. Uchiyama, M. Kuwahara, and Y. Takeda, Temporal response measurements of GaAs-Based photocathodes, Jpn. J. Appl. Phys. 52, 086401 (2013).

[31] S. Matsuba, Y. Honda, T. Miyajima, T. Uchiyama, M. Yamamoto, X. Jin, and Y. Takeda in Proceedings of the 3rd International Particle Accelerator Conference, New Orleans, 2012, edited by V. Suller (IEEE, Piscataway, 2012), p. 640, http://accelconf.web.cern.ch/AccelConf/ IPAC2012/papers/moppp035.pdf.

[32] S. Damelin and W. Miller, The Mathematics of Signal Processing (Cambridge University Press, Cambridge, England, 2011).

[33] M. Abramowitz and I. Stegun, Handbook on Mathematical Functions (Dover Publications, Inc., New York, 1965).

[34] MATLAB: The Language of Technical Computing by the MathWorks Inc., Natick, 2000.

[35] Y. Honda and T. Miyajima, in Proceedings of the 10th Annual Meeting of Particle Accelerator Society of Japan, Nagoya, 2013 (Particle Accelerator Society of Japan, Tokyo, 2013), p. 769 (in Japanese), https://www.pasj.jp/ web_publish/pasj10/proceedings/PDF/SAP1/SAP107.pdf.

[36] S. B. van der Geer and M. J. de Loos, The General Particle Tracer Code: Design, Implementation and Application (Technische Universiteit Eindhoven, Eindhoven, 2001), http://www.pulsar.nl/gpt/.

[37] T. Obina et al., in Proceedings of the 7th International Particle Accelerator Conference, Busan, 2016, edited by C. Petit-Jean-Genaz, D. E. Kim, K. S. Kim, I. S. Ko, K. R. Kim, and V. R. W. Schaa (JACoW, Geneva, 2016), p. 1835, http://accelconf.web.cern.ch/AccelConf/ipac2016/papers/ tupow036.pdf.

[38] E. Kako, S. Noguchi, T. Shishido, K. Watanabe, and Y. Yamamoto, in Proceedings of the 3rd International Particle Accelerator Conference, New Orleans, 2012, edited by V. Suller (IEEE, Piscataway, 2012), p. 2230, http:// accelconf.web.cern.ch/AccelConf/IPAC2012/papers/ weppc012.pdf.

[39] Y. Honda, T. Konomi, T. Obina, K. Umemori, H. Sakai, E. Kako, and T. Miyajima, in Proceedings of the 13th Annual Meeting of Particle Accelerator Society of Japan, Chiba, 2013 (Particle Accelerator Society of Japan, Tokyo, 2016), p. 893 (in Japanese), https://www.pasj.jp/web_publish/ pasj2016/proceedings/PDF/TUP0/TUP025.pdf.

[40] T. Wangler, Principles of RF Linear Accelerators (Wiley, New York, 1998).

[41] J.-G. Hwang, E.-S. Kim, T. Miyajima, Y. Honda, K. Harada, M. Shimada, R. Takai, T. Kume, S. Nagahashi, T. Obina et al., Analysis on effects of transverse electric field in an injector cavity of compact-ERL at KEK, Nucl. Instrum. Methods Phys. Res., Sect. A 753, 97 (2014). 
[42] A. Facco and V. Zvyagintsev, Beam steering in superconducting quarter-wave resonators: An analytical approach, Phys. Rev. ST Accel. Beams 14, 070101 (2011).

[43] R. Helm and R. Miller, in Linear Accelerators, edited by P. M. Lapostolle and A. L. Septier (North-Holland Publishing Company, Amsterdam and John Wiley \& Sons, New York, 1970), p. 115.

[44] A. Shishlo and J. Holmes, ORNL Report No. ORNL/TM2015/247, 2015, http://info.ornl.gov/sites/publications/ files/Pub55859.pdf.

[45] S. C. Hartman and J. B. Rosenzweig, Ponderomotive focusing in axisymmetric rf linacs, Phys. Rev. E 47, 2031 (1993).

[46] Z. Q. He, Z. Zheng, Z. Liu, Y. Zhang, and J. Wei, in Proceedings of the 26th Linear Accelerator Conference, Tel-Aviv, 2012, edited by M. Draper, C. Carli, J. Poole, L. Liljeby, and S. Lupu (JACoW, Geneva, 2013), p. 609, http://accelconf.web.cern.ch/AccelConf/LINAC2012/papers/ tupb058.pdf.

[47] J. Rosenzweig and L. Serafini, Transverse particle motion in radio-frequency linear accelerators, Phys. Rev. E 49, 1599 (1994).

[48] T. Menzel and H. K. Stokes, User's Guide for the POISSON/SUPERFISH Group of Codes (Los Alamos National Laboratory, Los Alamos, 1987).

[49] R. Strattan and F. Young, Fields in square helmholtz coils, Appl. Sci. Res. 9, 117 (1961).

[50] A. K. M. Alamgir, J. Fang, C. Gu, and Z. Han, Square Helmholtz coil with homogeneous field for magnetic measurement of longer HTS tapes, Physica (Amsterdam) 424, 17 (2005).

[51] M. Misakian, Equations for the magnetic field produced by one or more rectangular loops of wire in the same plane, J. Res. Natl. Inst. Stand. Technol. 105, 557 (2000).

[52] D. Herceg, A. Juhas, and M. Milutinov, A design of a four square coil system for a biomagnetic experiment, Ser. Elec. Energ. 22, 285 (2009).

[53] OPERA 3D Software for electro-magnetic design by Vector Fields Ltd., England, 1999.

[54] K. Harada, T. Kume, S. Nagahashi, N. Nakamura, S. Sakanaka, and A. Ueda, in Proceedings of the 6th International Particle Accelerator Conference, Richmond, 2015, edited by S. Henderson, T. Satogata, and V. R. W.
Schaa (JACoW, Geneva, 2015), p. 2899, http://accelconf .web.cern.ch/AccelConf/IPAC2015/papers/wepma055.pdf.

[55] A. Ueda, K. Harada, S. Nagahashi, T. Kume, M. Shimada, T. Miyajima, N. Nakamura, and K. Endo, in Proceedings of the 7th International Particle Accelerator Conference, Busan, 2016, edited by C. Petit-Jean-Genaz, D. E. Kim, K. S. Kim, I. S. Ko, K. R. Kim, and V. R. W. Schaa (JACoW, Geneva, 2016), p. 1111, http://accelconf.web .cern.ch/AccelConf/ipac2016/papers/tupmb005.pdf.

[56] P. Lorrain and D. Corson, Electromagnetism, Principles and Applications (W. H. Freeman \& Co, New York, 1990).

[57] M. Borland, APS Report No LS-287, 2000, http://www .aps.anl.gov/Accelerator_Systems_Division/Accelerator_ Operations_Physics/presentations/ICAP2000.pdf.

[58] L. Cultrera, I. Bazarov, A. Bartnik, B. Dunham, S. Karkare, R. Merluzzi, and M. Nichols, Thermal emittance and response time of a cesium antimonide photocathode, Appl. Phys. Lett. 99, 152110 (2011).

[59] T. Miyajima, in Proceedings of ERL17, Geneva, 2017 (CERN, Geneva, 2017), THIACC001_TALK, https://indico .cern.ch/event/470407/contributions/2610959/attachments/ 1481060/2296668/THIACC001_TALK.PPTX.

[60] N. Nishimori, in Proceedings of ERL17, Geneva, 2017 (CERN, Geneva, 2017), WEICCC001_TALK, https://indico .cern.ch/event/470407/contributions/2610931/attachments/ 1479885/2294375/WEICCC001_TALK.PPTX.

[61] M. Kurki, in Proceedings of the 8th International Particle Accelerator Conference, Copenhagen, 2017 (JACoW, Geneva, 2017), pp. 1440-1442.

[62] J. Wolberg, Data Analysis Using the Method of Least Squares: Extracting the Most Information from Experiments (Springer, Berlin, 2005).

[63] J. Lagarias, J. Reeds, M. Wright, and P. Wright, Convergence properties of the Nelder-Mead simplex algorithm in low dimensions, SIAM J. OPTIM. 9, 112 (1998).

[64] A. P. Prudnikov, Yu. A. Brychkov, and O. I. Marichev, Integrals and Series (Gordon \& Breach Science Publishers, New York, 1986), Vol. 2, pp. 343, 773, 788, and 793.

[65] E. Weber, Electromagnetic Theory (Dover, New York, 1965), p. 131. 